\title{
On the high frequency oscillatory tube flow of healthy human blood
}

\author{
Miguel A. Moyers-Gonzalez ${ }^{\mathrm{a}}$, Robert G. Owens ${ }^{\mathrm{b}}$, Jiannong Fang ${ }^{\mathrm{c}}$ \\ ${ }^{a}$ Department of Mathematical Sciences, University of Durham, Science Laboratories, South \\ Road, Durham DH1 3LE, UK \\ ${ }^{b}$ Département de mathématiques et de statistique, Université de Montréal, CP 6128 succ. \\ Centre-Ville, Montréal QC H3C 3J7, Canada \\ ${ }^{c}$ GEOLEP-ICARE-ENAC, Ecole Polytechnique Fédérale de Lausanne, 1015 Lausanne, \\ Switzerland
}

\begin{abstract}
In this paper pressure gradient vs. volume flow rate calculations over a wide range of oscillatory frequencies for oscillatory tube flow of healthy human blood are performed using the non-homogeneous hemorheological model of MoyersGonzalez et al. [25, 26, 27]. Results at low $(2 \mathrm{~Hz})$ oscillatory frequencies are shown to be in close conformity to the experimental data of Thurston [39] and the behaviour may be interpreted using a linear viscoelastic model. As the oscillatory frequencies increase a resonant frequency at which flow rate amplitude enhancement occurs is encountered. For frequencies greater than the resonant frequency the pressure gradient amplitude required to maintain a constant volume flow rate amplitude increases with the oscillatory frequency.

For very high frequency oscillations we use a multiple time scales technique in conjunction with our non-homogeneous hemorheological model to solve for the leading order flow variables. It is found that the leading order expressions for the cell number density, average aggregate size and $r r$-component of elastic stress (i.e. that due to the red blood cells) are functions only of the radial component $r$. The $O(1)$ elastic shear stress is shown to be zero, so that, for sufficiently large values of the oscillatory frequency, the red cell contribution to the total shear stress tends to zero. Using our multiple time scales method it is also shown that the model behaves in the very high frequency regime like a generalised linear viscoelastic fluid, having a radially dependent complex viscosity. This allows us to explain the computed results using asymptotic expressions for the in phase and $\pi / 2$ out of phase components of the pressure gradient in a linear viscoelastic fluid. In particular, we may predict the apparent complex viscosity of human blood in very high frequency oscillatory tube flow.
\end{abstract}

Key words: Non-homogeneous blood flow, high frequency oscillations, resonance, flow rate enhancement, multiple time scales

Email address: owens@dms.umontreal.ca (Robert G. Owens)

Preprint submitted to Elsevier

June 20, 2009 


\section{Introduction}

It is well known that when the frequency $\hat{\omega}$ of pressure gradient oscillations driving oscillatory tube flow of a Newtonian fluid gets very large the velocity field is not able to keep up with the rapidly changing pressure field (see, the mathematical details in chapter 4.11 of the monograph of [47], for example). A consequence of this is that if the pressure gradient amplitude is fixed at all frequencies, the peak velocity values will fall below the maximum of the velocity profile of the steady Poiseuille flow corresponding to $\hat{\omega}=0$. The problem of a linear Maxwell fluid oscillating in a tube was solved by Broer [6] and that of the general linear viscoelastic fluid by Fredrickson [17]. In a Newtonian fluid the volume flow rate will not be in phase at any frequency with the pressure gradient because of inertia. In the case of a linear viscoelastic fluid, however, there is an additional phase shift due to elasticity and this may, at a critical volume flow rate amplitude and frequency (conditions for "viscoelastic resonance" [39]), cancel the shift due to inertia, making the volume flow rate and the pressure gradient perfectly in phase (see, for example, Eqn. (18) and the discussion of the low flow rate amplitude experimental results of Thurston [38] for $P_{M}^{\prime \prime}$ in Fig. 1(b)). As shown in Section 3, a linear viscoelastic fluid may behave more and more like an elastic solid as the oscillatory frequency increases, meaning that the volume flow rate and the pressure gradient will tend to being $\pi / 2$ out of phase. As with a Newtonian fluid, the volume flow rate amplitude of a linear viscoelastic fluid will decrease to zero as $\hat{\omega} \rightarrow 0$ if the pressure gradient amplitude is fixed.

The dependence of the rheological properties of human blood on the frequency of oscillations in oscillatory tube flow has been investigated by a number of authors and some classic treatises are to be found in articles by Coulter and Singh [11], Kunz and Coulter [21] and Thurston [37, 38]. We may define, as did the authors of the first two articles just cited, a dynamic apparent viscosity for blood as being the viscosity of a Newtonian fluid which exhibits the same pressure gradient-volume flow rate under the same conditions of oscillatory flow. The dynamic apparent viscosity thus defined may therefore be considered as some average viscosity of the blood in a particular oscillatory flow. For each of the tubes used in the experiments of Coulter and Singh [11] and in an attempt to clearly distinguish frequency from shear-rate effects, the volume flow rate amplitude was fixed whilst allowing the oscillatory frequency to vary up to $10 \mathrm{~Hz}$. Under these circumstances, it was found that the dynamic apparent viscosity was an increasing function of frequency, and was a convincing demonstration of the non-Newtonian character of blood in this flow. This result was in contrast to the earlier findings of Kunz and Coulter [21] who reported that the dynamic apparent viscosity decreased with increasing oscillatory frequency. It should be borne in mind, however, that in the case of the experiments in [21] the stroke volume rather than the volume flow rate amplitude was fixed, thus resulting in a linear dependence of the latter on the oscillatory frequency. This had as one effect an increase in the average shear rate as the frequency increased and undoubtedly led to shear-thinning effects. The results of both sets of authors 
demonstrated that the hydraulic resistance (proportional to the part of the pressure gradient which is in phase with the volume flow rate) showed a dramatic difference from what could be predicted on the basis of the classical Newtonian theory of Womersley [45]. The quadrature ( $\pi / 2$ out of phase) component of the pressure gradient, however, was in close conformity with the linearized theory. These results were in agreement with the earlier experimental data of Fry et al. [18] who performed an in vivo study in the descending thoracic aorta of five dogs of the pressure gradient - blood velocity relationship.

In [37] Thurston presented experimental results for oscillatory flow of $50 \%$ hematocrit blood in a tube and showed that for each oscillatory frequency considered, a critical mean velocity gradient existed beyond which the relationship between the viscous and elastic components of the mean shearing stress and the mean velocity gradient ceased to be linear. Thereafter, maintaining the mean velocity gradient at levels below the critical values, the author investigated how the real and imaginary parts of the complex viscosity varied with frequency, since it was now reasonable to suppose that the rheological behaviour of the blood samples could be described by a linear viscoelastic model. Thurston [37] chose higher upper values of the frequencies than in the experiments of either Coulter and Singh [11] or Kunz and Coulter [21]. For frequencies less than about $0.1 \mathrm{~Hz}$ the viscous (real) part of the complex viscosity seemed to be constant and the imaginary part of the complex viscosity (related to the elastic modulus) was an increasing function of oscillation frequency. However, for much larger frequencies both components were decreasing functions of frequency. The experimentally determined complex viscosity of [37] was used by the same author some three years later in order to accurately predict using linear viscoelastic theory the magnitude and phase of the hydraulic impedance of blood undergoing oscillatory flow in a number of different diameter tubes, over a frequency range of $2 \mathrm{~Hz}$ to $200 \mathrm{~Hz}$. Again, all experiments were performed at mean shear rates kept sufficiently small that the fluid behaviour remained linear throughout. That in all cases the phase of the impedance passed from negative, at low frequency, to $\pi / 2$, at sufficiently high frequency, meant that the blood exhibited a viscous response at low frequencies, an elastic solid response at high frequencies and a viscoelastic response at intermediate values of the frequency. Re-expressed in terms of the pressure gradient - volume flow rate relationship, this means that at low frequency oscillations the pressure gradient may be expected to be approximately in phase with the volume flow rate but that, as the frequency gets very large the pressure gradient oscillations will now be in phase with the strain, and $\pi / 2$ out of phase with the volume flow rate.

The non-homogeneous blood model of Moyers-Gonzalez et al. [25, 26, 27] and its simpler homogeneous flow variant $[16,29]$ have been thoroughly validated against experimental data. In [29], Owens showed that close agreement existed between the predictions of the proposed homogeneous hemorheological model and the hysteretic shear stress data of [7] in a Couette viscometer. In the experiments of Bureau et al. the outer cylinder was accelerated and subsequently decelerated to produce a triangular variation of shear rate with time. Depending on the maximum shear rate attained and the timescales over which the entire 
experiment was performed, both elastic and thixotropic behaviour could be discerned from the resulting shear stress curves. Measurements by Thurston [38] of the in-phase and quadrature components of the pressure gradient amplitude as functions of the volume flow rate amplitude in oscillatory and pulsatile blood flow in rigid tubes have proved a valuable source of data for the validation of the time-dependent homogeneous and non-homogeneous models [27]. The simpler homogeneous version of the model $[16,29]$ had earlier been tested in a similar way but the improvement in the capacity of the non-homogenous model to accurately predict the experimental pressure gradient-volume flow rate data demonstrated the non-negligible role played by wall effects in a tubes of this size. The decrease of the ratio of the tube hematocrit to the discharge hematocrit as the diameter of the tube in which a blood sample flows decreases was first discovered by Robin Fåhraeus in 1929 [14] and is due to red cell migration from the tube walls. In [31] Pries et al. developed an empirical formula relating the tube hematocrit to the discharge hematocrit on the basis of data from experiments in which human red blood cell suspensions were perfused through glass tubes of different diameters [9, 14]. Two years later, Pries et al. [30] assembled the results of sixty years of steady flow viscosity measurements done with human, porcine and canine blood in capillary tubes in an attempt to elucidate the relationship that exists between the apparent viscosity and tube diameter (the so-called Fåhraeus-Lindqvist effect [15]). By using the empirical parametric description of the Fåhraeus effect from [31] Moyers-Gonzalez et al. [25] were able to accurately predict the apparent viscosity data curve presented in [30].

In our recent paper [27] on the modelling of oscillatory blood flow in a tube we observed that as the frequency of the (constant amplitude) pressure gradient oscillations increases

(1) the velocity field and shear stress continue to oscillate with the same frequency but with a frequency-dependent phase shift,

(2) the peak values of the velocity field and shear stress decrease,

(3) flow variables such as the red cell number density, average aggregate size and $r r$-component of the red cell contribution to the stress tensor tend to steady functions of the radial coordinate $r$.

It is the primary purpose of the present paper to provide a mathematical analysis of the equations of the non-homogeneous model of Moyers-Gonzalez et al. [25, 26,27 in the limit of high-frequency and to thus shed light on the observations (1) - (3) above. To accomplish this we employ the method of multiple time scales (see, for example, chapter 13 of [19]) and Section 4.2 and Appendix C, where we do this, form the mathematical core of the present article.

The outline of the paper is as follows: In Section 3 we present some of the established mathematical theory of axisymmetric oscillatory flow of a linear viscoelastic fluid in an infinitely long right circular cylindrical tube (see, for example, [36]). This material will later be used to interpret both the small flow rate amplitude and the high oscillatory frequency results of the blood flow simulations in Section 5. 
The linear viscoelastic theory is followed in Section 4.1 by a recapitulation of the governing equations of the non-homogeneous hemorheological model of Moyers-Gonzalez et al. [25, 27]. Unlike in the case of a linear viscoelastic fluid, no explicit expression is available directly from the constitutive equations and equation of linear momentum for the components of the pressure gradient that are in-phase and $\pi / 2$ out of phase with the volume flow rate. However, for a given pressure gradient amplitude and oscillatory frequency, we indicate how these two components may be numerically computed. In Section 4.2 we use a multiple time scales analysis in order to derive new simplified time-averaged differential equations for the macroscopic properties of blood undergoing rapid oscillations in a tube. The multiple time scales analysis relies, for simplicity and clarity, upon the assumption that the axial velocity field $v_{z}$ is sinusoidal. However, as is made plain in Section 2, it is an oscillatory pressure gradient

$$
-\frac{\partial p}{\partial z}=P_{M} \cos \hat{\omega} t,
$$

that is, in fact, prescribed. The justification for why, when volume flow rates are sufficiently small, the volume flow rate oscillates with the same frequency as the pressure gradient is simply that in this case the fluid behaves like a linear viscoelastic model. In Appendix $\mathrm{C}$ we show that $U$ also oscillates with approximately the same frequency $\hat{\omega}$ as $\partial p / \partial z$ when a modified Womersley number $W \gg 1$.

Finally, in Section 5 we demonstrate the excellent agreement that exists

(a) between our numerical predictions and the experimental results of Thurston [38] for the in-phase and $\pi / 2$ out of phase components of the pressure gradient as functions of the volume flow rate amplitude, at modest oscillatory frequencies,

and

(b) between the predictions of numerical simulations based on the time-averaged equations and the full set of model equations for high oscillatory frequencies.

\section{Oscillatory tube flow}

Consider the axisymmetric oscillatory flow of an incompressible fluid in an infinitely long right circular cylindrical tube. Let us introduce dimensionless cylindrical coordinates $\boldsymbol{x}=(r, \theta, z)$ and suppose that the tube has equation $r=1$. The fluid velocity is assumed to be of the form $\boldsymbol{v}=\left(v_{r}, v_{\theta}, v_{z}\right)=\left(0,0, v_{z}(r, t)\right)$. The $z$-component of the non-dimensional equation of linear momentum may then be written as

$$
R e \frac{\partial v_{z}}{\partial t}=-\frac{\partial p}{\partial z}+\frac{1}{r} \frac{\partial}{\partial r}\left(r T_{r z}\right),
$$


where $R e$ is a Reynolds number, $T_{r z}$ is the shear stress component of the extrastress tensor $\boldsymbol{T}$ and $p$ is the pressure. The total Cauchy stress tensor $\boldsymbol{\sigma}$ is defined as

$$
\boldsymbol{\sigma}:=-p \boldsymbol{\delta}+\boldsymbol{T},
$$

where $\boldsymbol{\delta}$ denotes the identity tensor, so that the right-hand side of (1) is just the $(r, z)$ component of the divergence of $\boldsymbol{\sigma}$.

Throughout this paper we suppose that the flow is driven by a sinusoidally varying pressure gradient of the form

$$
-\frac{\partial p}{\partial z}=P_{M} \cos \hat{\omega} t
$$

where $P_{M}$ denotes the pressure gradient amplitude and $\hat{\omega}$ is the dimensionless angular frequency. Suppose now that the volume flow rate

$$
U:=2 \pi \int_{r=0}^{1} v_{z} r d r
$$

is also sinusoidal, oscillating with the same frequency $\hat{\omega}$ as the pressure gradient, but with a possible phase shift. This is shown to be true in the case of a linear viscoelastic fluid in Section 3 and may therefore be assumed to be approximately true for the (nonlinear) blood model of Section 4, provided that the volume flow rate amplitude is sufficiently small. For a justification of this hypothesis in the case of $\hat{\omega} \gg 1$ we refer to the discussion in Appendix C. We may now write $U$ in complex form as

$$
U=U_{M}^{*} \exp (i \hat{\omega} t)
$$

where $U_{M}^{*}:=U_{M}^{\prime}+i U_{M}^{\prime \prime}$ is a complex amplitude and it is understood that the real part of the right-hand side should be taken. Evaluating the real part of the right-hand side of (4) we therefore have

$$
U=U_{M}^{\prime} \cos \hat{\omega} t-U_{M}^{\prime \prime} \sin \hat{\omega} t=U_{M} \cos (\hat{\omega} t+A),
$$

where now $U_{M}=\sqrt{U_{M}^{\prime 2}+U_{M}^{\prime \prime 2}}$ is the (real) volume flow rate amplitude and $A=\tan ^{-1}\left(U_{M}^{\prime \prime} / U_{M}^{\prime}\right)$ is the phase shift. Note that the pressure gradient (3) may also be represented in complex form as

$$
-\frac{\partial p}{\partial z}=P_{M}^{*} \exp (i(\hat{\omega} t+A))
$$

where the real and imaginary parts of the complex pressure gradient amplitude $P_{M}^{*}$, denoted by $P_{M}^{\prime}$ and $P_{M}^{\prime \prime}$, respectively, are given by

$$
P_{M}^{\prime}=P_{M} \cos A \text { and } P_{M}^{\prime \prime}=-P_{M} \sin A .
$$

\section{Oscillatory tube flow of a linear viscoelastic fluid}

Let us seek solutions to (1) of the (complex) form

$v_{z}=v_{z}^{*}(r) \exp (i \hat{\omega} t), T_{r z}=\eta^{*} \frac{\partial v_{z}}{\partial r}$ and $-\partial p / \partial z=P_{M}^{*} \exp (i(\hat{\omega} t+A))$, 
$v_{z}^{*}$ being a complex valued function and $\eta^{*}=\eta^{\prime}-i \eta^{\prime \prime}$ a dimensionless complex viscosity. Note that $v_{z}^{*}$ and the complex amplitude $U_{M}^{*}$ appearing in (4) are related through

$$
U_{M}^{*}:=2 \pi \int_{r=0}^{1} v_{z}^{*} r d r .
$$

Inserting the complex representations (8) into (1) and dividing throughout by $\exp (i \hat{\omega} t)$ yields the equation

$$
i \hat{\omega} R e v_{z}^{*}-\frac{\eta^{*}}{r} \frac{d}{d r}\left(r \frac{d v_{z}^{*}}{d r}\right)=P_{M}^{*} \exp (i A) .
$$

We may solve (10) for $v_{z}^{*}$ subject to a no-slip condition $v_{z}^{*}=0$ on the wall $r=1$ and $d v_{z}^{*} / d r=0$ on $r=0$. The resulting solution for the axial velocity $v_{z}$ is

$$
v_{z}=v_{z}^{*} \exp (i \hat{\omega} t)=\frac{i}{\hat{\omega} R e} P_{M}^{*} \exp (i A)\left(\frac{J_{0}(\kappa r)}{J_{0}(\kappa)}-1\right) \exp (i \hat{\omega} t),
$$

where $J_{0}$ denotes a zeroth order Bessel function of the first kind and $\kappa$ is a complex parameter defined by

$$
\kappa^{2}:=-\frac{i \hat{\omega} R e}{\eta^{*}} .
$$

We now integrate both sides of (10) over a unit disk and divide throughout by $2 \pi \exp (i A)$. This leads to the volume flow rate - pressure gradient amplitude relation

$$
U_{M}=-\frac{i \pi}{\hat{\omega} R e} P_{M}^{*}\left(1-\frac{2 J_{1}(\kappa)}{\kappa J_{0}(\kappa)}\right),
$$

where use has been made of the relation $U_{M}=U_{M}^{*} \exp (i A)$. The above developments generalize those of Womersley [44] who derived a relation analogous to (13) for a Newtonian fluid (in which case $\eta^{\prime}$ is just the fluid viscosity and $\left.\eta^{\prime \prime}=0\right)$.

The modulus of $\kappa$ (see (12)) may be interpreted as a modified Womersley number $W$ (cf. Eqn. (56) of [16]):

$$
W:=\sqrt{\frac{\hat{\omega} R e}{\left|\eta^{*}\right|}} .
$$

The Cox-Merz rule [12] may now be used to establish approximate upper and lower bounds on $W$ as follows:

$$
W_{a}<W<W_{b},
$$

where

$$
W_{a}^{2}:=\frac{\eta_{\infty}}{\left(\eta_{0}+\eta_{N}\right)} \frac{R e \hat{\omega}}{D e_{\infty}}, W_{b}^{2}:=\frac{\eta_{\infty}}{\left(\eta_{\infty}+\eta_{N}\right)} \frac{R e \hat{\omega}}{D e_{\infty}} .
$$


In (16) $\eta_{0}, \eta_{\infty}$ and $\eta_{N}$ denote, respectively, the zero shear-rate polymeric viscosity (i.e. due to the red cells), the infinite shear-rate polymeric viscosity and the Newtonian (plasma) viscosity (see Table 1). The bounds in (15) with $W_{a}$ and $W_{b}$ as defined in (16) come from the fact that the dimensionless infinite shear rate viscosity is $\left(\eta_{\infty}+\eta_{N}\right) D e_{\infty} / \eta_{\infty}$ and the dimensionless zero shear rate viscosity is $\left(\eta_{0}+\eta_{N}\right) D e_{\infty} / \eta_{\infty}$ so that the modulus of $\eta^{*}$ is between the two.

\section{1. $P_{M}^{*}-U_{M}$ relations when $W \ll 1$ and $W \gg 1$}

In [36], Thurston derived leading order expressions for $P_{M}^{\prime}$ and $P_{M}^{\prime \prime}$ from (13) for $W \ll 1$. In terms of our non-dimensional variables these translate into

$$
\begin{aligned}
& P_{M}^{\prime} \approx \frac{8 U_{M}}{\pi} \eta^{\prime}, \\
& P_{M}^{\prime \prime} \approx \frac{8 U_{M}}{\pi}\left(-\eta^{\prime \prime}+\frac{\hat{\omega} R e}{6}\right) .
\end{aligned}
$$

We note from (17)-(18) that, as commented on already in the Introduction, in the case of a Newtonian fluid the volume flow rate will not be in phase at any frequency with the pressure gradient because of inertia. However, in the case of a linear viscoelastic fluid there is an additional phase shift due to the presence of the $\eta^{\prime \prime}$ term in (18). Relations (17)-(18) will be used in Section 5 to interpret the results that we obtain for small flow rate amplitude, low frequency oscillations of blood in a tube. In this case $\eta^{*}$ should be interpreted as an apparent (spaceaveraged) complex viscosity, since, in general, the complex viscosity for small amplitude oscillations of blood in a tube is $r$-dependent.

To derive an asymptotic relation for very large Womersley numbers we begin by writing the complex viscosity in polar form, so that, as in [36],

$$
\eta^{*}=\eta^{\prime}-i \eta^{\prime \prime}=\left|\eta^{*}\right| e^{-i \varphi}
$$

where

$$
\left|\eta^{*}\right|=\sqrt{\eta^{\prime 2}+\eta^{\prime \prime 2}} \text { and } \tan \varphi=\eta^{\prime \prime} / \eta^{\prime} .
$$

We remark that $\varphi$ is just $\pi / 2$ minus the, so-called "loss angle" (see, for example, Eqn. (3.44) of [4]) and physically, we require that

$$
0 \leq \varphi \leq \pi / 2 \text {. }
$$

Small values of $\varphi$ indicate that the viscous component of the complex viscosity is very much more important than the elastic component. Values of $\varphi$ close to $\pi / 2$ mean that the opposite is true. Now if we take $\sqrt{-i}=e^{-i \pi / 4}$ then

$$
\kappa=\sqrt{\frac{\hat{\omega} R e}{\left|\eta^{*}\right|}} e^{i(\varphi / 2-\pi / 4)}=W e^{i(\varphi / 2-\pi / 4)} .
$$

From (20) we see that $-\pi / 4 \leq \varphi / 2-\pi / 4 \leq 0$. Therefore, the imaginary part of $\kappa=W \sin (\varphi / 2-\pi / 4) \leq 0$. For $\sin (\varphi / 2-\pi / 4)<0$ and $W \rightarrow \infty$ we get

$$
\frac{J_{1}(\kappa)}{J_{0}(\kappa)} \sim-i
$$


(Note the sign error in (17) of [36]). From Eqn. (13) we then get

$$
U_{M} \sim \frac{-i \pi}{\hat{\omega} R e} P_{M}^{*}\left(1+\frac{2 i}{\kappa}\right),
$$

which, inverting the formula, implies that

$$
P_{M}^{*} \sim \frac{\hat{\omega} R e}{-i \pi} U_{M}\left(1-\frac{2 i}{\kappa}\right)=\frac{\hat{\omega} R e}{\pi} U_{M}\left(i+\frac{2}{\kappa}\right) .
$$

Therefore, taking real and imaginary parts, expressions for $P_{M}^{\prime}$ and $P_{M}^{\prime \prime}$ are

$$
\begin{aligned}
P_{M}^{\prime} & \sim \frac{2 U_{M}}{\pi} \sqrt{\left|\eta^{*}\right| \hat{\omega} R e} \cos (\varphi / 2-\pi / 4), \\
P_{M}^{\prime \prime} & \sim U_{M}\left(\frac{\hat{\omega} R e}{\pi}-\frac{2}{\pi} \sqrt{\left|\eta^{*}\right| \hat{\omega} R e} \sin (\varphi / 2-\pi / 4)\right) .
\end{aligned}
$$

(Note that had we used $\sqrt{i}=-e^{-i \pi / 4}$ in the definition of $\kappa$ in (21), its imaginary part would have become positive and under these circumstances

$$
\frac{J_{1}(\kappa)}{J_{0}(\kappa)} \sim i
$$

so that the expressions (24)-(25) would remain unchanged).

$\left|\eta^{*}\right|$, the modulus of $\eta^{*}$, is expected to remain bounded for all $\hat{\omega}$ and, indeed, experimental evidence (see, for example, Fig. 7 of [39]) indicates that, for sufficiently high values of the frequency, it is a monotonic decreasing function of $\hat{\omega}$. We may infer from (24)-(25) that as the oscillation frequency increases sufficiently

i. the fluid behaves more and more like an elastic solid, in the sense that $P_{M}^{\prime} / P_{M}^{\prime \prime} \rightarrow 0$ as $\hat{\omega} \rightarrow \infty$

ii. the volume flow rate amplitude will decrease to zero if the pressure gradient amplitude $P_{M}$ is fixed.

\section{Oscillatory tube flow of human blood.}

In a series of recent papers Moyers-Gonzalez et al. [25, 26, 27] derived and used a new microstructure-based constitutive model for the prediction of non-homo-geneous flows of whole healthy human blood. As pointed out in the Introduction, this model and its simpler homogeneous flow variant $[16,29]$ have been thoroughly validated against experimental data for time-dependent Couette flow [7], steady non-homogeneous Poiseuille flow [30] and low frequency oscillatory and pulsatile tube flow [38].

The model is based upon the representation of red cell aggregates (the socalled rouleaux) by Hookean dumbbells. Each dumbbell has properties that depend upon the number of cells in the rouleaux and it may be stretched, 
transported and orientated by the surrounding fluid motion, as well as fragment into two smaller dumbbells or coalesce with another to represent a longer rouleau. Interactions between dumbbells are assumed to be binary and the rates of aggregation and fragmentation are given by experimentally-inspired functions of the local shear rate [28]. The interested reader is referred to the paper of Moyers-Gonzalez et al. [25] for a more detailed explanation of the kinetic theory underpinning the derivation of the governing equations of the model.

\subsection{Recapitulation of governing equations and calculation of $P_{M}^{\prime}$ and $P_{M}^{\prime \prime}$}

Let $N_{0}(\boldsymbol{x}, t)$ denote the number density of red cells at some point in the flow domain having position vector $\boldsymbol{x}$ and at some time $t$. If $M(\boldsymbol{x}, t)$ denotes the aggregate number density then $n(\boldsymbol{x}, t):=N_{0}(\boldsymbol{x}, t) / M(\boldsymbol{x}, t)$ is the average aggregate size. In the case of whole blood, the extra-stress $\boldsymbol{T}$ (see (1)-(2)) is the sum of a Newtonian and elastic contribution, the former coming from the plasma and the latter the ensemble of dumbbells. We thus write $\boldsymbol{T}$ in the form

$$
\boldsymbol{T}=\eta\left(\nabla \boldsymbol{v}+\nabla \boldsymbol{v}^{T}\right)+\boldsymbol{\tau}
$$

where $\eta$ denotes the dimensionless plasma viscosity and

$$
\boldsymbol{\tau}=\sum_{k=1}^{\infty} \boldsymbol{\tau}_{k}
$$

is the elastic stress tensor, calculated as the sum of the elastic stress tensors $\boldsymbol{\tau}_{k}$ that give the contribution to the Cauchy stress tensor from aggregates of size $k$ $(k=1,2,3, \ldots)$. We will henceforth refer to an aggregate of $k$ cells as a $k$-mer.

Suppose that the rate at which an $(i+j)$-mer is formed from the coalescence of an $i$-mer and a $j$-mer is given by the function $a=a(\dot{\gamma})$ where $\dot{\gamma}$ is the local shear rate. Suppose too that the function $b(\dot{\gamma})$ determines the rate at which

an $(i+j)$-mer fragments to form an $i$-mer and a $j$-mer. Then, the system of coupled convection-diffusion-reaction equations describing the evolution of the continuum variables $N_{0}, M, \boldsymbol{\tau}, \boldsymbol{v}$ and $p$ in the model of Moyers-Gonzalez et al. $[25,26,27]$ for axisymmetric tube flow is as follows:

$$
\begin{aligned}
& \frac{\partial N_{0}}{\partial t}-\frac{1}{P e} \frac{1}{r} \frac{\partial}{\partial r}\left(r \frac{\partial N_{0}}{\partial r}\right)+\frac{1}{P e} \frac{1}{r} \frac{\partial}{\partial r}\left(r \frac{\partial \tau_{r r}}{\partial r}\right)=0 \\
& \frac{\partial M}{\partial t}-\frac{1}{P e} \frac{1}{r} \frac{\partial}{\partial r}\left(r \frac{\partial M}{\partial r}\right)+\frac{1}{P e} \frac{1}{r} \frac{\partial}{\partial r}\left(r \frac{\partial \sigma_{r r}}{\partial r}\right)+\frac{a N_{0}}{2 n} M-\frac{b}{2}\left(N_{0}-M\right)=0 \\
& D e \frac{\partial \tau_{r r}}{\partial t}-\frac{D e}{P e}\left(\frac{2}{r} \frac{\partial}{\partial r}\left(r \frac{\partial \tau_{r r}}{\partial r}\right)\right)+\tau_{r r}=0 \\
& D e \frac{\partial \tau_{r z}}{\partial t}-\frac{D e}{P e}\left(\frac{1}{r} \frac{\partial}{\partial r}\left(r \frac{\partial \tau_{r z}}{\partial r}\right)-\frac{\tau_{r z}}{r^{2}}\right)-\left(\tau_{r r}+N_{0}\right) D e \frac{\partial v_{z}}{\partial r}+\tau_{r z}=0
\end{aligned}
$$




$$
\begin{aligned}
& D e \frac{\partial \tau_{z z}}{\partial t}-\frac{D e}{P e}\left(\frac{1}{r} \frac{\partial}{\partial r}\left(r \frac{\partial \tau_{z z}}{\partial r}\right)+\frac{1}{r} \frac{\partial}{\partial r}\left(r \frac{\partial \tau_{r r}}{\partial r}\right)\right)-2 D e \frac{\partial v_{z}}{\partial r} \tau_{r z}+\tau_{z z}=0 \\
& D e \frac{\partial \sigma_{r r}}{\partial t}-\frac{D e}{P e}\left(\frac{2}{r} \frac{\partial}{\partial r}\left(r \frac{\partial \sigma_{r r}}{\partial r}\right)\right)+\sigma_{r r}=0 \\
& R e \frac{\partial v_{z}}{\partial t}-\eta \frac{1}{r} \frac{\partial}{\partial r}\left(r \frac{\partial v_{z}}{\partial r}\right)-\frac{1}{r} \frac{\partial}{\partial r}\left(r \tau_{r z}\right)+\frac{\partial p}{\partial z}=0
\end{aligned}
$$

In (29) and (33) $\sigma_{r r}$ denotes the $r r$-component of the second-order tensor $\boldsymbol{\sigma}$, defined as

$$
\boldsymbol{\sigma}:=\sum_{k=1}^{\infty} \frac{\tau_{k}}{k}
$$

The fragmentation rate function $b(\dot{\gamma})$, appearing in $(29)$, is expressed in terms of the aggregation rate $a$ according to the relation that holds in a homogeneous flow:

$$
b:=\frac{a N_{0, h o m}}{n_{s t}\left(n_{s t}-1\right)},
$$

where $n_{s t}$ is the average rouleau size and $N_{0, \text { hom }}$ is the (constant) number density in steady homogeneous flow. Let us introduce the dimensionless fragmentation rate coefficient

$$
g_{n}(\dot{\gamma}):=a(\dot{\gamma}) M+b(\dot{\gamma}) \frac{(n-1)}{2} .
$$

Then, in (30)-(33) the (shear rate and time-dependent) Deborah number De (a non-dimensional relaxation time of the fluid) is defined as

$$
D e:=\frac{n D e_{\infty}}{1+g_{n} n D e_{\infty}},
$$

where $D e_{\infty}$ is a (constant, dimensionless) Maxwellian relaxation time, to which $D e$ tends as $\dot{\gamma} \rightarrow \infty$. The Péclet number $P e$, appearing in Eqn.s (28)-(33) relates the rate of convection of the flow to the rate of mass diffusion. The boundary layer at $r=1$ was shown by Moyers-Gonzalez and Owens [26] to be of thickness $O\left(\left(D e_{\infty} / P e\right)^{1 / 2}\right)$. For details of the physical parameters that require prescription and of the values that they have been assigned in this paper we refer the reader to the beginning of Section 5 and to Table 1.

At this point we note from (28)-(34) that in order to find the other field variables we have no need of the elastic normal stress component $\tau_{z z}$. Therefore, in order to determine the $P_{M}^{\prime}$ and $P_{M}^{\prime \prime}$ predicted by the non-homogeneous blood model described above, $P_{M}$ and $\hat{\omega}$ are first prescribed in (3) and then the system of equations (28)-(31) and (33)-(34) is solved numerically subject to suitable initial and boundary conditions. These are supplied in Appendix A. Once periodic conditions have been established, the phase difference $A$ between the volume flow rate and the pressure gradient may be measured. The in phase and $\pi / 2$ out of phase components of the pressure gradient amplitude are then 
found from (7). It is expected that such a procedure will work in two limiting cases:

(a). When the volume flow rate amplitude $U_{M} \ll 1$. In this case the model is expected to behave as a linear viscoelastic fluid.

(b). When the angular oscillatory frequency $\hat{\omega} \gg 1$. For a justification in this case, see the discussion in Appendix C.

4.2. A multiple time scales analysis of (28)-(30) and (33)-(34) for high-frequency oscillatory tube flow

We now turn our attention to the behaviour of the non-homogeneous blood model in high frequency oscillatory tube flow. To this end, we employ a multiple time scales analysis of the governing model equations (28)-(34). Considerable simplifications may be brought to bear on our approach firstly, by noting, as we did above, that $\tau_{z z}$ is not required for the computation of the other kinematic and stress variables and second, and more importantly, by assuming an oscillatory axial velocity profile,

$$
v_{z}=\hat{v}_{z}(r) \exp (i \hat{\omega} t)
$$

Since, as explained above, it is the pressure gradient and not the velocity field that is prescribed, however, we simply assume for the moment that $\partial p / \partial z$ may be chosen in (34) in such a way that the corresponding axial velocity profile is of the form given by (39). An explanation of how, at least for $\hat{\omega}$ sufficiently large, a velocity profile of the form (39) may be expected from the imposition of an oscillatory pressure gradient is deferred to Appendix C.

We now define a fast time scale $\vartheta=\hat{\omega} t$ and seek multiple time scale expansions for $N_{0}, \tau_{r r}, M, \sigma_{r r}$ and $D e$ as follows:

$$
\begin{aligned}
N_{0} & =N_{0}^{(0)}(r, t, \vartheta)+\varepsilon N_{0}^{(1)}(r, t, \vartheta)+\varepsilon^{2} N_{0}^{(2)}(r, t, \vartheta)+\ldots, \\
\tau_{r r} & =\tau_{r r}^{(0)}(r, t, \vartheta)+\varepsilon \tau_{r r}^{(1)}(r, t, \vartheta)+\varepsilon^{2} \tau_{r r}^{(2)}(r, t, \vartheta)+\ldots, \\
M & =M^{(0)}(r, t, \vartheta)+\varepsilon M^{(1)}(r, t, \vartheta)+\varepsilon^{2} M^{(2)}(r, t, \vartheta)+\ldots, \\
\sigma_{r r} & =\sigma_{r r}^{(0)}(r, t, \vartheta)+\varepsilon \sigma_{r r}^{(1)}(r, t, \vartheta)+\varepsilon^{2} \sigma_{r r}^{(2)}(r, t, \vartheta)+\ldots, \\
D e & =D e^{(0)}(r, t, \vartheta)+\varepsilon D e^{(1)}(r, t, \vartheta)+\varepsilon^{2} D e^{(2)}(r, t, \vartheta)+\ldots,
\end{aligned}
$$

where $\varepsilon:=\hat{\omega}^{-1}$. In (44) the Deborah function $D e$ is defined as

$$
D e=\frac{n D e_{\infty}}{1+g_{n} n D e_{\infty}} .
$$

To take account of the fact that all dependent variables now depend upon a fast time $(\vartheta)$ and a slow time $(t)$, the partial time derivative in each of (28)-(34) is rewritten in the form

$$
\frac{\partial}{\partial t} \rightarrow \frac{\partial}{\partial t}+\hat{\omega} \frac{\partial}{\partial \vartheta}=\frac{\partial}{\partial t}+\frac{1}{\varepsilon} \frac{\partial}{\partial \vartheta} .
$$




\subsection{1. $\tau_{r r}$}

From (41), (44) and (46) we see that (30) may be rewritten as

$$
\begin{aligned}
\left(D e^{(0)}+\varepsilon D e^{(1)}+\varepsilon^{2} D e^{(2)}+\ldots\right) & {\left[\left(\frac{\partial \tau_{r r}^{(0)}}{\partial t}+\frac{1}{\varepsilon} \frac{\partial \tau_{r r}^{(0)}}{\partial \vartheta}\right)+\varepsilon\left(\frac{\partial \tau_{r r}^{(1)}}{\partial t}+\frac{1}{\varepsilon} \frac{\partial \tau_{r r}^{(1)}}{\partial \vartheta}\right)\right.} \\
& \left.+\varepsilon^{2}\left(\frac{\partial \tau_{r r}^{(2)}}{\partial t}+\frac{1}{\varepsilon} \frac{\partial \tau_{r r}^{(2)}}{\partial \vartheta}\right)+\ldots\right] \\
-\left(D e^{(0)}+\varepsilon D e^{(1)}+\varepsilon^{2} D e^{(2)}+\ldots\right) \frac{1}{P e} \frac{2}{r} & \frac{\partial}{\partial r}\left(r\left(\frac{\partial \tau_{r r}^{(0)}}{\partial r}+\varepsilon \frac{\partial \tau_{r r}^{(1)}}{\partial r}+\varepsilon^{2} \frac{\partial \tau_{r r}^{(2)}}{\partial r}+\ldots\right)\right) \\
& +\left(\tau_{r r}^{(0)}+\varepsilon \tau_{r r}^{(1)}+\varepsilon^{2} \tau_{r r}^{(2)}+\ldots\right)=0 .
\end{aligned}
$$

Let's equate all $O\left(\varepsilon^{-1}\right), O(1)$, etc. terms in (47) to zero, which gives

$$
\begin{aligned}
& \left(O\left(\varepsilon^{-1}\right)\right): \quad D e^{(0)} \frac{\partial \tau_{r r}^{(0)}}{\partial \vartheta}=0 \Rightarrow \frac{\partial \tau_{r r}^{(0)}}{\partial \vartheta}=0 \\
& (O(1)): \quad D e^{(0)} \frac{\partial \tau_{r r}^{(0)}}{\partial t}+D e^{(1)} \frac{\partial \tau_{r r}^{(0)}}{\partial \vartheta}+D e^{(0)} \frac{\partial \tau_{r r}^{(1)}}{\partial \vartheta}-\frac{D e^{(0)}}{P e} \frac{2}{r} \frac{\partial}{\partial r}\left(\begin{array}{r}
\left.r \frac{\partial \tau_{r r}^{(0)}}{\partial r}\right) \\
+\tau_{r r}^{(0)}=0 .
\end{array}\right.
\end{aligned}
$$

4.2.2. $N_{0}$

Using (40), (41) and (46), Eqn. (28) is transformed to

$$
\begin{aligned}
\left(\frac{\partial N_{0}^{(0)}}{\partial t}+\frac{1}{\varepsilon} \frac{\partial N_{0}^{(0)}}{\partial \vartheta}\right) & +\varepsilon\left(\frac{\partial N_{0}^{(1)}}{\partial t}+\frac{1}{\varepsilon} \frac{\partial N_{0}^{(1)}}{\partial \vartheta}\right)+\varepsilon^{2}\left(\frac{\partial N_{0}^{(2)}}{\partial t}+\frac{1}{\varepsilon} \frac{\partial N_{0}^{(2)}}{\partial \vartheta}\right)+\ldots \\
& -\frac{1}{P e} \frac{1}{r} \frac{\partial}{\partial r}\left(r\left(\frac{\partial N_{0}^{(0)}}{\partial r}+\varepsilon \frac{\partial N_{0}^{(1)}}{\partial r}+\varepsilon^{2} \frac{\partial N_{0}^{(2)}}{\partial r}+\ldots\right)\right) \\
& +\frac{1}{P e} \frac{1}{r} \frac{\partial}{\partial r}\left(r\left(\frac{\partial \tau_{r r}^{(0)}}{\partial r}+\varepsilon \frac{\partial \tau_{r r}^{(1)}}{\partial r}+\varepsilon^{2} \frac{\partial \tau_{r r}^{(2)}}{\partial r}+\ldots\right)\right)=0 .
\end{aligned}
$$

As before, we now equate all $O\left(\varepsilon^{-1}\right), O(1)$, etc. terms in (50) to zero, which gives

$$
\begin{aligned}
& \left(O\left(\varepsilon^{-1}\right)\right): \quad \frac{\partial N_{0}^{(0)}}{\partial \vartheta}=0 \Rightarrow N_{0}^{(0)}=N_{0}^{(0)}(r, t) \\
& (O(1)): \quad \frac{\partial N_{0}^{(0)}}{\partial t}+\frac{\partial N_{0}^{(1)}}{\partial \vartheta}-\frac{1}{P e} \frac{1}{r} \frac{\partial}{\partial r}\left(r \frac{\partial N_{0}^{(0)}}{\partial r}\right)+\frac{1}{P e} \frac{1}{r} \frac{\partial}{\partial r}\left(r \frac{\partial \tau_{r r}^{(0)}}{\partial r}\right)=0
\end{aligned}
$$


From (52) we solve for $N_{0}^{(1)}$ to get

$N_{0}^{(1)}=\int\left(-\frac{\partial N_{0}^{(0)}}{\partial t}+\frac{1}{P e} \frac{1}{r} \frac{\partial}{\partial r}\left(r \frac{\partial N_{0}^{(0)}}{\partial r}\right)-\frac{1}{P e} \frac{1}{r} \frac{\partial}{\partial r}\left(r \frac{\partial \tau_{r r}^{(0)}}{\partial r}\right)\right) d \vartheta+f_{1}(r, t)$,

for some function $f_{1}$ of $r$ and $t$. Since, from (48) and (51) $\tau_{r r}^{(0)}$ and $N_{0}^{(0)}$ are both independent of $\vartheta$, the solution (53) becomes

$$
N_{0}^{(1)}=\left(-\frac{\partial N_{0}^{(0)}}{\partial t}+\frac{1}{P e} \frac{1}{r} \frac{\partial}{\partial r}\left(r \frac{\partial N_{0}^{(0)}}{\partial r}\right)-\frac{1}{P e} \frac{1}{r} \frac{\partial}{\partial r}\left(r \frac{\partial \tau_{r r}^{(0)}}{\partial r}\right)\right) \vartheta+f_{1}(r, t) .
$$

We set the secular term on the righthand side of (54) to zero (otherwise we have unbounded growth of $N_{0}^{(1)}$ as $\left.\vartheta \rightarrow \infty\right)$, which gives

$$
\frac{\partial N_{0}^{(0)}}{\partial t}-\frac{1}{P e} \frac{1}{r} \frac{\partial}{\partial r}\left(r \frac{\partial N_{0}^{(0)}}{\partial r}\right)+\frac{1}{P e} \frac{1}{r} \frac{\partial}{\partial r}\left(r \frac{\partial \tau_{r r}^{(0)}}{\partial r}\right)=0
$$

4.2.3. $\sigma_{r r}$

In an entirely analogous manner to the procedure followed for $\tau_{r r}$, we obtain

$$
\begin{aligned}
& \frac{\partial \sigma_{r r}^{(0)}}{\partial \vartheta}=0 \Rightarrow \sigma_{r r}^{(0)}=\sigma_{r r}^{(0)}(r, t), \\
& D e^{(0)} \frac{\partial \sigma_{r r}^{(0)}}{\partial t}+D e^{(1)} \frac{\partial \sigma_{r r}^{(0)}}{\partial \vartheta}+D e^{(0)} \frac{\partial \sigma_{r r}^{(1)}}{\partial \vartheta}-\frac{D e^{(0)}}{P e} \frac{2}{r} \frac{\partial}{\partial r}\left(r \frac{\partial \sigma_{r r}^{(0)}}{\partial r}\right)+\sigma_{r r}^{(0)}=0 .
\end{aligned}
$$

\subsection{4. $M$}

We adopt a similar approach to that used in Section 4.2.2 for the solution of Eqn. (29). Since the aggregation and disaggregation rate functions $a$ and $b$ depend only upon the local shear rate $\dot{\gamma}$, it follows from $(39)$ that $a=a(r, \vartheta)$ and $b=b(r, \vartheta)$. To avoid the notation becoming unduly cumbersome, we indicate explicitly in what follows, however, only the dependence of these two functions on $\vartheta$. Using (40),(42) and (43) allows us to write down

$$
\begin{aligned}
& \left(\frac{\partial M^{(0)}}{\partial t}+\frac{1}{\varepsilon} \frac{\partial M^{(0)}}{\partial \vartheta}\right)+\varepsilon\left(\frac{\partial M^{(1)}}{\partial t}+\frac{1}{\varepsilon} \frac{\partial M^{(1)}}{\partial \vartheta}\right)+\varepsilon^{2}\left(\frac{\partial M^{(2)}}{\partial t}+\frac{1}{\varepsilon} \frac{\partial M^{(2)}}{\partial \vartheta}\right)+\ldots \\
& -\frac{1}{P e} \frac{1}{r} \frac{\partial}{\partial r}\left(r\left(\frac{\partial M^{(0)}}{\partial r}+\varepsilon \frac{\partial M^{(1)}}{\partial r}+\varepsilon^{2} \frac{\partial M^{(2)}}{\partial r}+\ldots\right)\right) \\
& +\frac{1}{P e} \frac{1}{r} \frac{\partial}{\partial r}\left(r\left(\frac{\partial \sigma_{r r}^{(0)}}{\partial r}+\varepsilon \frac{\partial \sigma_{r r}^{(1)}}{\partial r}+\varepsilon^{2} \frac{\partial \sigma_{r r}^{(2)}}{\partial r}+\ldots\right)\right) \\
& +\frac{a(\vartheta)}{2}\left(M^{(0)}+\varepsilon M^{(1)}+\varepsilon^{2} M^{(2)}+\ldots\right)^{2} \\
& -\frac{b(\vartheta)}{2}\left(\left(N_{0}^{(0)}-M^{(0)}\right)+\varepsilon\left(N_{0}^{(1)}-M^{(1)}\right)+\varepsilon^{2}\left(N_{0}^{(2)}-M^{(2)}\right)+\ldots\right)=0 . \quad \text { (58) }
\end{aligned}
$$


By setting the sum of terms of $O\left(\varepsilon^{-1}\right), O(1)$ etc. to zero we see that

$$
\begin{aligned}
\left(O\left(\varepsilon^{-1}\right)\right): \quad \frac{\partial M^{(0)}}{\partial \vartheta}=0 \Rightarrow & M^{(0)}=M^{(0)}(r, t) \\
(O(1)): \quad \frac{\partial M^{(0)}}{\partial t}+\frac{\partial M^{(1)}}{\partial \vartheta} & -\frac{1}{P e} \frac{1}{r} \frac{\partial}{\partial r}\left(r \frac{\partial M^{(0)}}{\partial r}\right)+\frac{1}{P e} \frac{1}{r} \frac{\partial}{\partial r}\left(r \frac{\partial \sigma_{r r}^{(0)}}{\partial r}\right) \\
& +\frac{a(\vartheta)}{2}\left(M^{(0)}\right)^{2}-\frac{b(\vartheta)}{2}\left(N^{(0)}-M^{(0)}\right)=0
\end{aligned}
$$

Let us define the function $R(r, t, \vartheta)$ as

$$
\begin{array}{r}
R:=\frac{\partial M^{(0)}}{\partial t}-\frac{1}{P e} \frac{1}{r} \frac{\partial}{\partial r}\left(r \frac{\partial M^{(0)}}{\partial r}\right)+\frac{1}{P e} \frac{1}{r} \frac{\partial}{\partial r}\left(r \frac{\partial \sigma_{r r}^{(0)}}{\partial r}\right) \\
+\frac{a(\vartheta)}{2}\left(M^{(0)}\right)^{2}-\frac{b(\vartheta)}{2}\left(N^{(0)}-M^{(0)}\right),
\end{array}
$$

and write this function in the following way:

$$
R=\langle R\rangle+H
$$

where the average over one cycle $\langle\cdot\rangle$ is defined as

$$
\langle\cdot\rangle:=\frac{1}{2 \pi} \int_{o}^{2 \pi} \cdot d \vartheta
$$

Then, solving (60) for $M^{(1)}$ and noting that $\langle R\rangle$ is independent of $\vartheta$ gives

$$
M^{(1)}=-\langle R\rangle \vartheta-\int H d \vartheta+f_{2}(r, t),
$$

for some function $f_{2}$ of $r$ and $t$. Eqn. (64) allows us to conclude (since the secular term must be zero) that

$$
\begin{aligned}
\frac{\partial M^{(0)}}{\partial t}-\frac{1}{P e} \frac{1}{r} \frac{\partial}{\partial r}\left(r \frac{\partial M^{(0)}}{\partial r}\right) & +\frac{1}{P e} \frac{1}{r} \frac{\partial}{\partial r}\left(r \frac{\partial \sigma_{r r}^{(0)}}{\partial r}\right) \\
& +\left(M^{(0)}\right)^{2} \frac{\langle a\rangle}{2}-\left(N_{0}^{(0)}-M^{(0)}\right) \frac{\langle b\rangle}{2}=0 .
\end{aligned}
$$

$D e^{(0)}$ is, in general, a function of $r, t$ and $\vartheta$. From (49) let us define

$$
S:=\frac{\partial \tau_{r r}^{(0)}}{\partial t}-\frac{1}{P e} \frac{2}{r} \frac{\partial}{\partial r}\left(r \frac{\partial \tau_{r r}^{(0)}}{\partial r}\right)+\frac{1}{D e^{(0)}} \tau_{r r}^{(0)} .
$$

Then, requiring that $\tau_{r r}^{(1)}$ be a bounded function of $\vartheta$, and following the same line of reasoning that allowed us to conclude that $\langle R\rangle=0$ (see the argument leading up to $(65)$ ), we write down $\langle S\rangle=0$. This means that $\tau_{r r}^{(0)}$ satisfies

$$
\frac{\partial \tau_{r r}^{(0)}}{\partial t}-\frac{1}{P e} \frac{2}{r} \frac{\partial}{\partial r}\left(r \frac{\partial \tau_{r r}^{(0)}}{\partial r}\right)+\left\langle\frac{1}{D e^{(0)}}\right\rangle \tau_{r r}^{(0)}=0 .
$$


By analogy, $\sigma_{r r}^{(0)}$ satisfies

$$
\frac{\partial \sigma_{r r}^{(0)}}{\partial t}-\frac{1}{P e} \frac{2}{r} \frac{\partial}{\partial r}\left(r \frac{\partial \sigma_{r r}^{(0)}}{\partial r}\right)+\left\langle\frac{1}{D e^{(0)}}\right\rangle \sigma_{r r}^{(0)}=0 .
$$

Eqn.s (55), (65), (67) and (68) may be solved subject to the boundary conditions and the non-local condition (100) supplied in Appendix A. It follows that if (55), (65), (67) and (68) supplemented with these conditions forms a well-posed system of equations, then the solutions will depend only upon $r$. In the next Section we investigate the linear stability of the time-dependent equations for $N_{0}^{(0)}, M^{(0)}, \tau_{r r}^{(0)}$ and $\sigma_{r r}^{(0)}$.

\subsection{Linear stability of (55), (65), (67) and (68)}

We now take a closer look at the $O(1)$ equations (55), (65), (67) and (68) for $N_{0}^{(0)}, M^{(0)}, \tau_{r r}^{(0)}$ and $\sigma_{r r}^{(0)}$, respectively, and, in particular, examine the linear stability of their solutions about the steady solutions $N_{s}, M_{s}, \tau_{s}$ and $\sigma_{s}$ to the equations

$$
\begin{gathered}
-\frac{1}{P e} \frac{2}{r} \frac{d}{d r}\left(r \frac{d \tau_{s}}{d r}\right)+\left\langle\frac{1}{D e_{s}}\right\rangle \tau_{s}=0 \\
-\frac{1}{P e} \frac{2}{r} \frac{d}{d r}\left(r \frac{d \sigma_{s}}{d r}\right)+\left\langle\frac{1}{D e_{s}}\right\rangle \sigma_{s}=0 \\
-\frac{1}{P e} \frac{1}{r} \frac{d}{d r}\left(r \frac{d M_{s}}{\partial r}\right)+\frac{1}{P e} \frac{1}{r} \frac{d}{d r}\left(r \frac{d \sigma_{s}}{d r}\right) \\
+\left(M_{s}\right)^{2} \frac{\langle a\rangle}{2}-\left(N_{s}-M_{s}\right) \frac{\langle b\rangle}{2}=0
\end{gathered}
$$

and

$$
-\frac{1}{P e} \frac{1}{r} \frac{d}{d r}\left(r \frac{d N_{s}}{d r}\right)+\frac{1}{P e} \frac{1}{r} \frac{d}{d r}\left(r \frac{d \tau_{s}}{d r}\right)=0 .
$$

As shown in Section 2.2.4 of [25], $N_{s}$ and $\tau_{s}$ must satisfy the simple linear relation

$$
N_{s}=\tau_{s}+C,
$$

where the constant $C$ is chosen so that the integral condition (100) holds. The averaged steady zeroth order reciprocal Deborah number $\left\langle D e_{s}^{-1}\right\rangle$ appearing in (69)-(70) is defined as

$$
\left\langle\frac{1}{D e_{s}}\right\rangle:=\frac{M_{s}^{2}+\left(\langle b\rangle N_{s}\left(N_{s}-M_{s}\right) / 2+\langle a\rangle N_{s} M_{s}^{2}\right) D e_{\infty}}{N_{s} M_{s} D e_{\infty}} .
$$


Suppose that $N_{0}^{(0)}, M^{(0)}, \tau_{r r}^{(0)}$ and $\sigma_{r r}^{(0)}$ may be written as small, time $t$-dependent perturbations about $N_{s}, M_{s}, \tau_{s}$ and $\sigma_{s}$ of the form

$$
\begin{aligned}
N_{0}^{(0)} & =N_{s}+\epsilon N_{1}^{(0)}:=N_{s}+\epsilon \hat{N}(r) \exp (\lambda t), \\
M^{(0)} & =M_{s}+\epsilon M_{1}^{(0)}:=M_{s}+\epsilon \hat{M}(r) \exp (\lambda t), \\
\tau_{r r}^{(0)} & =\tau_{s}+\epsilon \tau_{1}^{(0)}:=\tau_{s}+\epsilon \hat{\vartheta}(r) \exp (\lambda t), \\
\sigma_{r r}^{(0)} & =\sigma_{s}+\epsilon \sigma_{1}^{(0)}:=\sigma_{s}+\epsilon \hat{\sigma}(r) \exp (\lambda t),
\end{aligned}
$$

where $|\epsilon| \ll 1$ and each of the perturbations has been supposed to be a function of $r$ alone multiplied by $\exp (\lambda t)$ for some parameter $\lambda$. The consequence of the perturbations (75)-(78) upon the Deborah number $D e^{(0)}$ is that it too is perturbed and we write

$$
D e^{(0)}=D e_{s}+\epsilon D e_{1}+O\left(\epsilon^{2}\right):=D e_{s}+\epsilon \widehat{D e}(r, \vartheta) \exp (\lambda t)+O\left(\epsilon^{2}\right) .
$$

In Appendix B we derive an expression for $\widehat{D e}$ in terms of $\hat{N}, \hat{M}, N_{s}$ and $M_{s}$. Substituting each of (75)-(78) into Eqns. (55), (65), (67) and (68) and neglecting all terms of $O\left(\epsilon^{2}\right)$ may now be shown to lead to the system of equations for $(\hat{N}, \hat{\vartheta}, \hat{M}, \hat{\sigma})$ :

$$
\begin{aligned}
& \lambda \hat{N}-\frac{1}{P e} \frac{1}{r} \frac{d}{d r}\left(r \frac{d \hat{N}}{d r}\right)+\frac{1}{2}\left(\left(\lambda+\left\langle\frac{1}{D e_{s}}\right\rangle\right) \hat{\vartheta}-(\alpha \hat{N}+\beta \hat{M}) \tau_{s}\right)=0, \\
& \left(\lambda+\left\langle\frac{1}{D e_{s}}\right\rangle\right) \hat{\vartheta}-\frac{1}{P e} \frac{2}{r} \frac{d}{d r}\left(r \frac{d \hat{\vartheta}}{d r}\right)-(\alpha \hat{N}+\beta \hat{M}) \tau_{s}=0 \\
& \lambda \hat{M}-\frac{1}{P e} \frac{1}{r} \frac{d}{d r}\left(r \frac{d \hat{M}}{d r}\right)+\frac{1}{2}\left(\left(\lambda+\left\langle\frac{1}{D e_{s}}\right\rangle\right) \hat{\sigma}-(\alpha \hat{N}+\beta \hat{M}) \sigma_{s}\right) \\
& \quad+M_{s} \hat{M}\langle a\rangle-(\hat{N}-\hat{M}) \frac{\langle b\rangle}{2}=0 \\
& \left(\lambda+\left\langle\frac{1}{D e_{s}}\right\rangle\right) \hat{\sigma}-\frac{1}{P e} \frac{2}{r} \frac{d}{d r}\left(r \frac{d \hat{\sigma}}{d r}\right)-(\alpha \hat{N}+\beta \hat{M}) \sigma_{s}=0
\end{aligned}
$$

where the functions $\alpha$ and $\beta$ are defined in Eqns. (111)-(112). The system (80)-(83) may be written in more compact form as

$$
\mathcal{L} \boldsymbol{v} \equiv \frac{2}{P e} \frac{1}{r} \frac{d}{d r}\left(r \frac{d \boldsymbol{v}}{d r}\right)=(\lambda \boldsymbol{A}+\boldsymbol{C}) \boldsymbol{v},
$$


where $\boldsymbol{v}=(\hat{N}, \hat{\vartheta}, \hat{M}, \hat{\sigma})^{T}$ and the matrices $\boldsymbol{A}$ and $\boldsymbol{C}$ are given by

$$
\begin{aligned}
\boldsymbol{A} & =\left(\begin{array}{cccc}
2 & 1 & 0 & 0 \\
0 & 1 & 0 & 0 \\
0 & 0 & 2 & 1 \\
0 & 0 & 0 & 1
\end{array}\right), \\
\boldsymbol{C} & =\left(\begin{array}{cccc}
-\alpha \tau_{s} & \left\langle D e_{s}^{-1}\right\rangle & -\beta \tau_{s} & \\
-\alpha \tau_{s} & \left\langle D e_{s}^{-1}\right\rangle & -\beta \tau_{s} & 0 \\
\left(-\alpha \sigma_{s}-\langle b\rangle\right) & 0 & \left.-\beta \sigma_{s}+2\langle a\rangle M_{s}+\langle b\rangle\right) & \left\langle D e_{s}^{-1}\right. \\
-\alpha \sigma_{s} & 0 & -\beta \sigma_{s} & \left\langle D e_{s}^{-1}\right\rangle
\end{array}\right) .
\end{aligned}
$$

Now suppose that $\boldsymbol{P}$ is a transition matrix effecting the diagonalization of the matrix $\boldsymbol{A}$. Then, introducing a change of variables $\boldsymbol{v}=\boldsymbol{P} \boldsymbol{w}$ the generalized eigenvalue problem (84) may be rewritten in the form

$$
\frac{2}{P e} \frac{1}{r} \frac{d}{d r}\left(r \frac{d \boldsymbol{w}}{d r}\right)=\left(\lambda \operatorname{diag}(2,1,2,1)+\boldsymbol{P}^{-1} \boldsymbol{C P}\right) \boldsymbol{w}
$$

so that (84) may be seen to be a perturbation of a self-adjoint vector SturmLiouville problem. Unfortunately, $\boldsymbol{P}^{-1} \boldsymbol{C P}$ is both a complicated function of $r$ and non-symmetric, so that we resort to numerical methods to approximate the eigenvalues $\lambda$ of (84). Details are supplied in Section 5.3.

\section{Results}

\subsection{Choice of parameters}

The physical parameters that require prescribing in order to use our nonhomogeneous model are the plasma viscosity $\eta_{N}$, the infinite shear rate viscosity due to the red cells $\eta_{\infty}$, the characteristic relaxation time $\lambda_{H}$ for a single red cell,

the infinite shear rate Deborah number $D e_{\infty}$ (see (38) defined as the product of $\lambda_{H}$ and a characteristic shear rate, the tube radius $R$, a characteristic cell length $\ell_{0}$ and, finally, the fluid density $\rho$. The values of these parameters are supplied in Table 1. All values, except for $D e_{\infty}$ in Section 5.3, are the same as in [27]. For details of how the aggregation and fragmentation functions $a(\dot{\gamma})$ and $b(\dot{\gamma})$ are chosen, see Appendix A of [27]. Once these parameters have been chosen, the manner in which the non-dimensionalization of the governing equations is done means that the Reynolds number $R e$, Péclet number $P e$ and dimensionless viscosity $\eta$, appearing in (28)-(34) are defined as

$$
R e:=\frac{\rho R^{2} D e_{\infty}^{2}}{\lambda_{H} \eta_{\infty}}, P e:=\left(\frac{24 D e_{\infty}}{\ell_{0}^{2}}\right) R^{2}, \eta:=\frac{\eta_{N} D e_{\infty}}{\eta_{\infty}} .
$$

5.2. $P_{M}^{\prime}$ and $P_{M}^{\prime \prime}$ computations for $\omega \in\left[4 \pi \mathrm{rads}^{-1}, 400 \pi \mathrm{rads}^{-1}\right]$

In this Section we will perform and interpret numerical computations of the in-phase and $\pi / 2$ out of phase components of the pressure gradient amplitude, $P_{M}^{\prime}$ and $P_{M}^{\prime \prime}$ as functions of both the frequency and the volume flow rate 


\begin{tabular}{lcccc}
\hline Parameter & symbol & value & units \\
\hline fluid density & $\rho$ & 1053.6 & $\mathrm{kgm}^{-3}$ \\
plasma viscosity & $\eta_{N}$ & 0.001 & Pa.s \\
zero shear rate polymeric viscosity & $\eta_{0}$ & 0.0326 & Pa.s \\
high shear rate polymeric viscosity & $\eta_{\infty}$ & & 0.003 & Pa.s \\
Maxwell relaxation time & $\lambda_{H}$ & 0.005 & $\mathrm{~s}$ \\
infinite shear rate Deborah number & $D e_{\infty}$ & $1.4(\S 5.2), 1.0(\S 5.3)$ & - \\
tube radius & $R$ & $430(\S 5.2), 100(\S 5.3)$ & $\mu m$ \\
characteristic cell length & $\ell_{0}$ & & 13.8 & $\mu m$ \\
\hline
\end{tabular}

Table 1: Material and flow parameters and their assigned values

amplitude. Physiological values of the angular frequency of pulsatile flow in the arteries of a healthy human adult at rest would typically be in the range of $2 \pi \mathrm{rad} s^{-1}$ to $2.67 \pi \mathrm{rad} s^{-1}$ ( 60 to 80 heart beats per minute), although in a healthy twenty year old engaging in vigorous exercise this could go as high as 200 beats per minute $\left(6.67 \pi \mathrm{rads}^{-1}\right)$ [1]. The range of angular frequencies considered in our numerical experiments therefore includes physiological values but goes far beyond these in an effort to better understand the rheology of blood at very high frequency oscillations. So that we can compare our results with those of the experiments of Thurston [38] we use physical (dimensional) values of these variables and display our results in terms of the root mean-square (rms) values (cyclical averages) of $P_{M}^{\prime}, P_{M}^{\prime \prime}$ and $U_{M}$. In the case of $U_{M}$ rms, for example, this is calculated in terms of $U_{M}$ as follows:

$$
U_{M} \mathrm{rms}:=\sqrt{\frac{1}{T} \int_{0}^{T} U_{M}^{2} \cos ^{2}\left(\frac{2 \pi t}{T}\right) d t}=\frac{1}{\sqrt{2}} U_{M},
$$

where $T$ is the period of oscillation. Likewise, $P^{\prime} \mathrm{rms}=P_{M}^{\prime} / \sqrt{2}$ and $P^{\prime \prime} \mathrm{rms}$ $=P_{M}^{\prime \prime} / \sqrt{2}$. We define

$$
P_{M} \mathrm{rms}:=\sqrt{P^{\prime} \mathrm{rms}^{2}+P^{\prime \prime} \mathrm{rms}^{2}}
$$

so that the relationship between the rms values of the components of the pressure gradient amplitude and $P_{M}$ rms is the same as in Eqn. (7). The physical frequency $\omega$ is related to the dimensionless value $\hat{\omega}$ via $\omega=\hat{\omega} D e_{\infty} / \lambda_{H}$. All numerical results for the non-homogeneous model were obtained with a secondorder finite element method and a simple fractional time step method, as outlined in Section 3 of an earlier paper [27].

Fig. 1 shows how $\left|P^{\prime}\right| \mathrm{rms},\left|P^{\prime \prime}\right| \mathrm{rms}$ and $P_{M}$ rms vary as functions of $U_{M} \mathrm{rms}$ over a range of frequencies varying from $\omega=4 \pi \mathrm{rad} s^{-1}$ to $400 \pi \mathrm{rad} s^{-1}$. At the lowest frequency we compare the theoretical predictions with the experimental data of Thurston [38]. The agreement is seen to be excellent. At this frequency, we see from Fig. 2(a) that the phase shift $A$ passes from being positive to negative as the volume flow rate increases, and is equal to zero at $U_{M} \mathrm{rms} \approx$ 
$1.35 \times 10^{-9} \mathrm{~m}^{3} \mathrm{~s}^{-1}$. The same phenomenon may be seen from the surface plot, Fig. 2(b) where, for small pressure gradient magnitudes and low frequencies $A>0$. This corresponds to viscoelastic behaviour: the aggregates are largely intact and may be deformed elastically. As the pressure gradient amplitude (and therefore the volume flow rate amplitude) increases, the microstructure is increasingly broken up and the fluid response becomes viscous. Referring to (7) we see that although $P^{\prime}$ rms remains positive over the whole range of volume flow rates considered here, $P^{\prime \prime} \mathrm{rms}$ is negative for $U_{M} \mathrm{rms} \lesssim 1.35 \times 10^{-9} \mathrm{~m}^{3} \mathrm{~s}^{-1}$ and positive otherwise. Thus, the local "minimum" visible in the graph of $\left|P^{\prime \prime}\right| \mathrm{rms}$ is, in fact, the crossover point of $P^{\prime \prime} \mathrm{rms}$ from negative to positive values.

Since volume flow rates in the vicinity of $1.35 \times 10^{-9} \mathrm{~m}^{3} \mathrm{~s}^{-1}$ are small (corresponding to dimensionless volume flow rates around $0.0606 \ll 1$ ), the fluid may be considered to be approximately a linear viscoelastic fluid with the complex viscosity $\eta^{*}$ in (8) now interpreted as an apparent (space-averaged, in some sense) complex viscosity. To convince ourselves that the modified Womersley number $W$ in (14) is small for the $\omega=4 \pi \mathrm{rad}^{-1}$ results shown in Figs. 1(a)-(c) we use the bounds in (15). In this case the bounds on the modified Womersley number are $W_{a}=0.2699$ and $W_{b}=0.7823$. Let us now refer to (17) and (18) to shed further light upon the behaviour of $P^{\prime} \mathrm{rms}$ and $P^{\prime \prime} \mathrm{rms}$ at $\omega=4 \pi \mathrm{rad} s^{-1}$ in Figs. 1 (a) and (b). $P^{\prime}$ rms is expected to be approximately a linear function of $U_{M} \mathrm{rms}$ for $|A| \ll 1$ with a positive slope and this translates into saying that the dynamic viscosity $\eta^{\prime}$ (representing the viscous contribution to $\eta^{*}$ ) is positive and insensitive to small changes in $U_{M} \mathrm{rms}$ near the point where $P^{\prime \prime}$ rms changes sign. By looking at (18) we understand the sign change in $P^{\prime \prime} \mathrm{rms}$ for values of $U_{M} \mathrm{rms}$ in the same range to indicate that the elastic contribution $\eta^{\prime \prime}$ to the complex viscosity decreases in magnitude, allowing the inertial terms $\hat{\omega} R e / 6$ to become more important for $U_{M} \mathrm{rms} \gtrsim 1.35 \times 10^{-9} \mathrm{~m}^{3} \mathrm{~s}^{-1}$.

From Fig. 1(b) we see that the crossover point (coinciding with $A=0$, and where the curve seems to have a minimum) on the $\left|P^{\prime \prime}\right|$ rms curve corresponding to $\omega=40 \pi \mathrm{rad} s^{-1}$ occurs at a smaller value of $U_{M} \mathrm{rms}$ than when $\omega=4 \pi \mathrm{rad} s^{-1}$. This is in keeping with the position of the last data point on Fig. 2(a), this latter Fig. predicting that had we chosen to plot $P^{\prime \prime}$ rms in Fig. 1(b) for intermediate values of $\omega$ we would have seen a right shift (increasing $U_{M} \mathrm{rms}$ along the $A=0$ curve) in the minimum in $\left|P^{\prime \prime}\right|$ rms before this moved left again. This nonmonotonic behaviour may, from (18), be seen to be associated with an increase in the magnitude of $\eta^{\prime \prime}$, indicating that at low volume flow rates blood becomes more elastic with small increases in oscillatory frequency. Referring now to the surface plot Fig. 2 (b) we see that the right and then left shift of the minimum in the graphs of $\left|P^{\prime \prime}\right| \mathrm{rms}$ for $\omega \in\left[4 \pi \mathrm{rad} s^{-1}, 40 \pi \mathrm{rad} s^{-1}\right]$ is associated with an increase followed by a decrease in the phase shift $A$ with increasing $\omega$ in the top left-hand (small $P_{M}$ rms-small $\omega$ ) corner of the figure.

Undoubtedly related to the observations above is the non-monotonic behaviour for sufficiently small values of $\omega$ of the rms pressure gradient amplitude $P_{M}$ rms. From Fig. 1(c) it may be seen that in order to maintain a given volume flow rate amplitude the required value of $P_{M}$ rms first decreases and then increases with $\omega$. Otherwise stated, if we were to fix the pressure gradi- 
ent the volume flow rate amplitude would first increase and then decrease with increasing oscillatory frequency. The frequency $\omega_{r}$ (say) where the maximum occurs may be viewed as a resonant frequency. The phenomena of resonant frequencies and flow enhancement in oscillatory pipe flow is well known in both the experimental and theoretical viscoelastic literature and further discussion of these may be found, for example, in [2, 8, 13, 32, 41, 46]. A helpful review of earlier work is to be found in the article of Siginer [35]. The analyses in these articles indicate that were we to produce plots of $P_{M} \mathrm{rms}$ against $U_{M} \mathrm{rms}$ for many more values of $\omega$ and for different size tubes then, depending upon the tube radius, multiple resonant frequencies might be in evidence. For the present tube $(R=430 \mu m)$ it appears that there is only one resonant frequency $\omega_{r}$ and that for $\omega>\omega_{r} P_{M}$ rms is an increasing function of $\omega$ when $U_{M}$ rms is fixed. This is to be expected, since, as shown in Fig. 3(a)-(b), the peak axial velocity values decrease (and with them, the volume flow rate amplitude) as $\omega$ increases beyond the resonant value. Thus, to maintain a given, fixed value of the volume flow rate with growing $\omega>\omega_{r}$ the pressure gradient amplitude has to be increased.

Resonance as described above occurs due to the coupling between the elastic nature of the fluid and the tube geometry [8]. The same behaviour may occur even in oscillatory flows of linear viscoelastic fluids [2] and, indeed, may be predicted from Eqn. (13) by plotting the modulus of $U_{M} / P_{M}^{*}$ against $\hat{\omega}$ and taking the complex viscosity $\eta^{*}$ to be that of a single mode linear Maxwell fluid, for example. The asymptotic expressions (24)-(25) for $P_{M}^{\prime}$ and $P_{M}^{\prime \prime}$ for a linear viscoelastic fluid would then predict that at sufficiently high frequencies the pressure gradient amplitude would need to be increased with increasing $\omega$ just to maintain the volume flow rate amplitude. This last conclusion is by no means unique to viscoelastic fluids: the volume flow rate amplitude in the oscillatory tube flow of a Newtonian fluid may be shown to decrease with oscillatory frequency if the pressure gradient amplitude is held fixed [47].

In Appendix $\mathrm{C}$ we show, using our non-homogeneous hemorheological model, that high frequency oscillatory pipe flow of healthy human blood may be described using the equation of motion (114) of a generalised linear viscoelastic model. We use the adjective "generalised" because the leading order complex viscosity is shown to be a function of $r$, rather than being a constant. It is therefore legitimate to interpret the pressure gradient-volume flow rate results that we obtain using our model using the relations (24)-(25) provided that both the modified Womersley number (14) and the oscillatory frequency $\hat{\omega}$ are large. Since the relations (24)-(25) are for a (constant viscosity) linear viscoelastic model, however, the complex viscosity appearing in these equations should be considered to be an apparent complex viscosity for the blood model i.e. an $r$ - averaged value. By numerically calculating values of $P^{\prime}$ rms and $P^{\prime \prime}$ rms at particular values of the rms volume flow rate amplitude $U_{M}$ rms and of the oscillatory frequency $\omega$ we may then divide the dimensional forms of (24)-(25) to compute $\tan (\varphi / 2-\pi / 4)$ and hence obtain $\varphi$. The phase angle $A$ (see $(7))$ is just the arctangent of the ratio of $-P^{\prime \prime}$ rms and $P^{\prime}$ rms. Eqn.s (24)-(25) lead us to conclude that as $\omega \rightarrow \infty$, the ratio $P_{M}^{\prime} / P_{M}^{\prime \prime} \rightarrow 0$ so that the phase angle $A$ 
decreases towards $-\pi / 2$.

For the non-homogeneous model we may deduce from (126) that for $\hat{\omega} \gg 1$ the polar angle $\varphi$ in (19) may be bounded approximately as follows:

$$
0 \leq \varphi \lesssim \arctan \left(\frac{2 \varepsilon}{\eta} \max _{0 \leq r \leq 1}\left(N_{s}(r)-N_{s}(1)\right)\right),
$$

so that, in particular $\varphi \rightarrow 0$ as $\hat{\omega} \rightarrow \infty$, since (for physical reasons) the leading order cell number density $N_{s}$ is a bounded function. Accurate computations of $P^{\prime} \mathrm{rms}$ and $P^{\prime \prime} \mathrm{rms}$ at large values of $\omega$ are extremely difficult due to the requirement of using highly refined meshes near the wall and taking very small time steps. Using a second-order finite element method (see above) with a maximum dimensionless time step of $\Delta t=0.001$ in order to compute non-homogeneous results at $\omega=400 \pi \mathrm{rad} s^{-1}, 800 \pi \mathrm{rad}^{-1}$ and $1000 \pi \mathrm{rad} s^{-1}$ we obtain the results shown in the first three lines of Table 2. From the approximate bounds (15) we estimate that the modified Womersley numbers for the three cases lie in the intervals $[2.7,7.82],[3.81,11.06]$ and $[4.27,12.37]$. With $\omega=400 \pi$ the upper bound in (90) equals 0.4568 so that from Table 2 the computed value of $\varphi$ at $\omega=400 \pi$ is seen to be well within the theoretical bounds. Similarly, the computed values of $\varphi$ at $\omega=800 \pi$ and at $\omega=1000 \pi$ fall within the bounds predicted by (90). $A$ is seen to be a monotonic decreasing function of $\omega$ and for all values of $\omega, A>-\pi / 2$.

More convincing results in verification of (24)-(25) and (124) are more easily possible with the homogeneous blood model $[16,29]$. One reason for this is because in Eqn. (124) $\tau_{r r}^{(0)}=0$ and $N_{0}^{(0)}=1$ so that the leading order components of the complex viscosity are independent of $r$. From (126) we therefore expect, as the frequency $\hat{\omega}$ grows to be very large, that the polar angle $\varphi$ will behave asymptotically as

$$
\varphi \sim \arctan \left(\frac{1}{\hat{\omega} \eta}\right) .
$$

We further expect that the agreement between the results for $\varphi$ computed from (24)-(25) and predicted by the asymptotic result (91) will get better and better with increasing $\omega$ (for $\omega$ sufficiently large), tube radius $R$ and pressure gradient amplitude, the latter quantities being important because of their influence on the modified Womersley number $W$. The homogeneous model results in the last column of Table 2 were computed using a second order finite difference method with a non-uniform grid and smallest step size $\Delta r=8 \times 10^{-5}$. Time stepping was achieved using an adaptive Runge-Kutta method, as described in [16]. Minimum modified Womersley numbers for these calculations, using (15), were $62.77,88.77,108.73$ and 153.76 and the corresponding values of the dimensionless oscillatory frequency $\hat{\omega}$ were $4.49,8.98,13.46$ and 26.93 . The results of the finite difference computations displayed in the table compare extremely well with the predictions $0.4455,0.2387,0.1592$ and 0.079 coming from (91). The percentage relative difference between the calculated value of $\varphi$ at $\omega=400 \pi \mathrm{rad}^{-1}$ and the corresponding asymptotic value is $1.85 \%$ and this has shrunk yet further to $1.04 \%$ by the time we compute the percentage relative 


\begin{tabular}{lcccccc}
\hline $\mathrm{H} / \mathrm{NH}$ & $\omega\left(\mathrm{rad} s^{-1}\right)$ & $U_{M} \mathrm{rms}\left(\mathrm{m}^{3} \mathrm{~s}^{-1}\right)$ & $P^{\prime} \mathrm{rms}\left(\mathrm{Nm}^{-3}\right)$ & $P^{\prime \prime} \mathrm{rms}\left(\mathrm{Nm}^{-3}\right)$ & $A(\mathrm{rad})$ & $\varphi(\mathrm{rad})$ \\
\hline $\mathrm{NH}$ & $400 \pi$ & $6.3521 \times 10^{-10}$ & 141.3072 & $1.5701 \times 10^{3}$ & -1.4810 & 0.1445 \\
$\mathrm{NH}$ & $800 \pi$ & $3.2417 \times 10^{-10}$ & 106.0427 & $1.5728 \times 10^{3}$ & -1.5035 & 0.1088 \\
$\mathrm{NH}$ & $1000 \pi$ & $2.6147 \times 10^{-10}$ & 88.3893 & $1.5739 \times 10^{3}$ & -1.5147 & 0.0511 \\
$\mathrm{H}$ & $400 \pi$ & $1.6725 \times 10^{-7}$ & 3.4989 & 707.0981 & -1.565848 & 0.437206 \\
$\mathrm{H}$ & $800 \pi$ & $8.3686 \times 10^{-8}$ & 2.1972 & 707.1034 & -1.567689 & 0.235094 \\
$\mathrm{H}$ & $1200 \pi$ & $5.5812 \times 10^{-8}$ & 1.7218 & 707.1047 & -1.568361 & 0.159644 \\
$\mathrm{H}$ & $2400 \pi$ & $2.7921 \times 10^{-8}$ & 1.1682 & 707.1058 & -1.569144 & 0.080236 \\
& & & & & & \\
\hline
\end{tabular}

Table 2: Phase and polar angles $A$ and $\varphi$ for frequencies $\omega \in\left[400 \pi \mathrm{rad} s^{-1}, 2400 \pi \mathrm{rad} s^{-1}\right]$. NH: Non-homogeneous model [25, 26, 27], tube radius $R=4.3 \times 10^{-4} \mathrm{~m}$, pressure gradient amplitude $P_{M}=2229.7 \mathrm{Nm}^{-3}$. H: Homogeneous model $[16,29]$, tube radius $R=1 \times 10^{-2} \mathrm{~m}$, pressure gradient amplitude $P_{M}=1000 \mathrm{Nm}^{-3}$.

difference for $\omega=2400 \pi \mathrm{rad} s^{-1}$. Note, however, that even if there were no numerical error at all in the calculations (an impossibility!) we would not expect to get exactly the value predicted by (91) since this is an asymptotic result. The gradual decrease of $A$ towards $-\pi / 2$ with increasing oscillatory frequency is to be seen from the sixth column of Table 2 and the consequence is that, as remarked on in the Introduction, the fluid behaves increasingly like an elastic solid in the sense that the volume flow rate oscillations tend towards being $\pi / 2$ out of phase with those of the applied pressure gradient. In fact, what is happening is that the inertial term $\hat{\omega} R e / \pi$ dominates over all others in (24)-(25) so that, despite the fact that the elastic component $\eta^{\prime \prime}$ tends to zero and the fluid therefore becomes increasingly Newtonian, the stress is proportional to the strain rather than to the strain rate.

\subsection{Multiple time scales}

In order to investigate the long term growth of infinitesimal perturbations to the steady leading order equation set (69)-(70) the system

$$
(\mathcal{L}-\boldsymbol{C}) \boldsymbol{v}=\lambda \boldsymbol{A v},
$$

(see (84)) was discretized using a Chebyshev collocation method (see, for example, $[23,24,34])$ and the resulting generalized eigenvalue problem was solved using the MATLAB routine eig.

We note here that even though spectral methods have enjoyed much use for the discretisation of eigenvalue problems, it is well known in the case of secondorder spectral differentiation operators, for example, that only a fraction $2 / \pi$ of the eigenvalues of the continuous problem can be approximated accurately $[42,43]$. Since it is only the dominant normal mode that interests us, however, this is not anticipated to be problematic in the present case.

Fig. 4 shows the dominant computed eigenvalue of the generalized eigenvalue problem (92) for values of the tube radius $R=50 \mu \mathrm{m}, 100 \mu \mathrm{m}$ and $200 \mu \mathrm{m}$, 


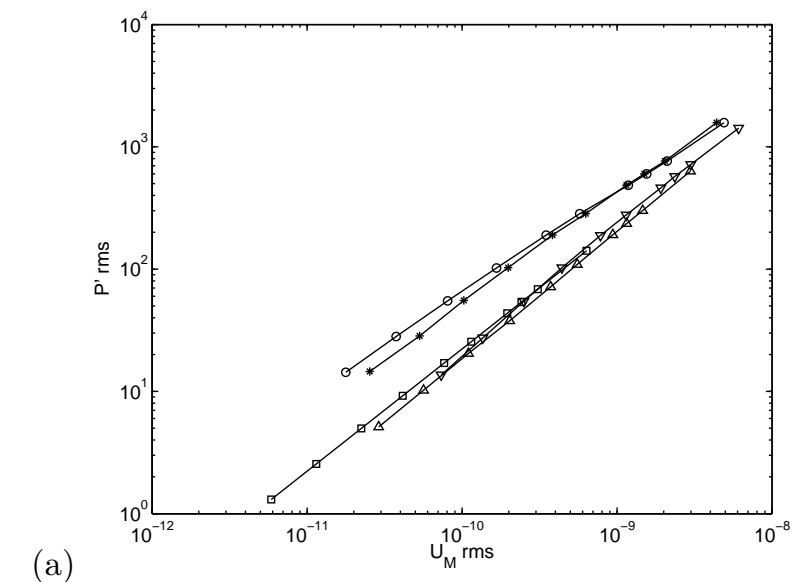

(a)

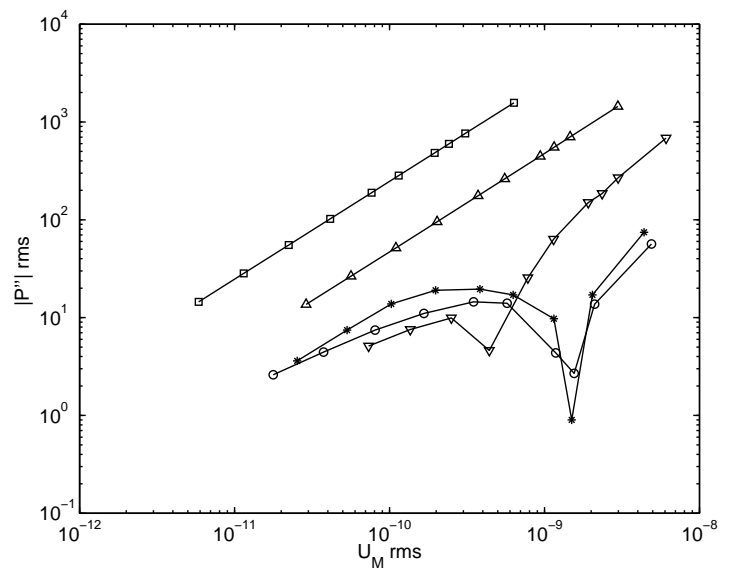

(b)

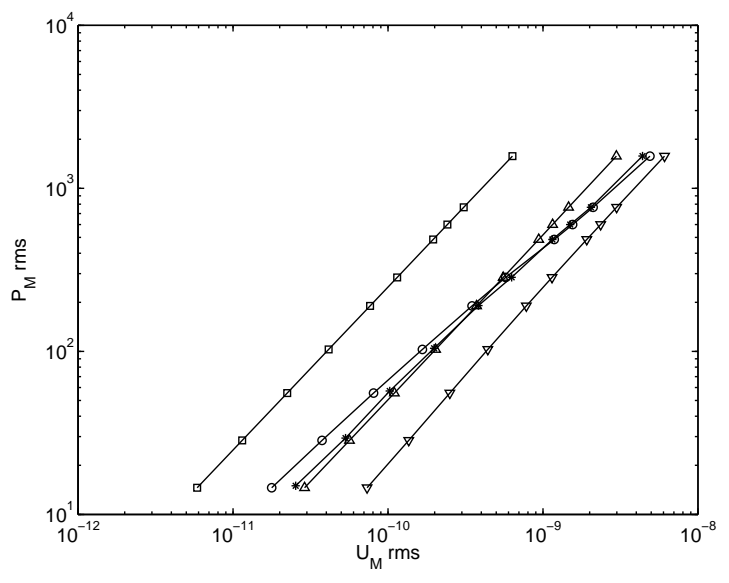

Figure 1: $R=430 \mu m$. (a) $\left|P^{\prime}\right| \operatorname{rms}\left(\mathrm{Nm}^{-3}\right)$, (b) $\left|P^{\prime \prime}\right| \mathrm{rms}\left(\mathrm{Nm}^{-3}\right)$ and (c) $P_{M} \mathrm{rms}\left(\mathrm{Nm}^{-3}\right)$ against $U_{M} \mathrm{rms}\left(m^{3} s^{-1}\right)$. ०: $\omega=4 \pi \mathrm{rads}^{-1}, *: \omega=4 \pi \mathrm{rads}^{-1}$, Thurston's [38] data, $\nabla$ : $\omega=40 \pi \mathrm{rad} s^{-1}, \Delta: \omega=80 \pi \mathrm{rad} s^{-1}, \square: \omega=400 \pi \mathrm{rad} s^{-1}$. 


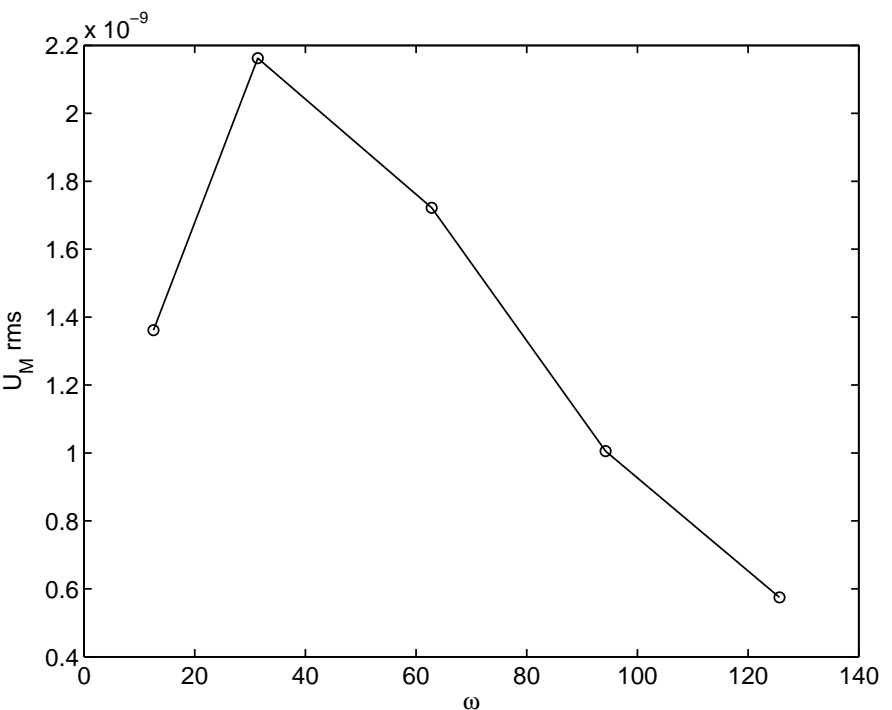

(a)

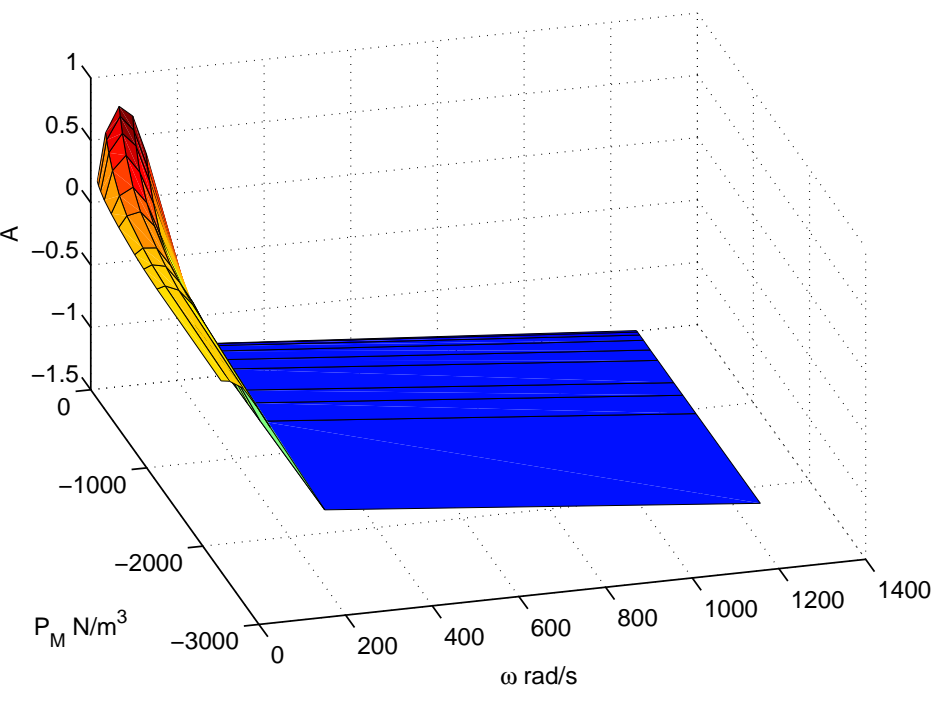

Figure 2: $R=430 \mu m$. Phase shift $A$. (a) $A=0$ contour plot in $\omega\left(\operatorname{rad} s^{-1}\right)-U_{M} \mathrm{rms}\left(m^{3} s^{-1}\right)$ space, (b) surface plot of $A$ in $\omega-P_{M}$ space. The data points shown in (a) correspond to $\omega=4 \pi \mathrm{rad} s^{-1}, 10 \pi \mathrm{rad} s^{-1}, 20 \pi \mathrm{rad} s^{-1}, 30 \pi \mathrm{rad} s^{-1}$ and $40 \pi \mathrm{rad} s^{-1}$ with $A>0$ below the curve and $A<0$ above it. 
(a)
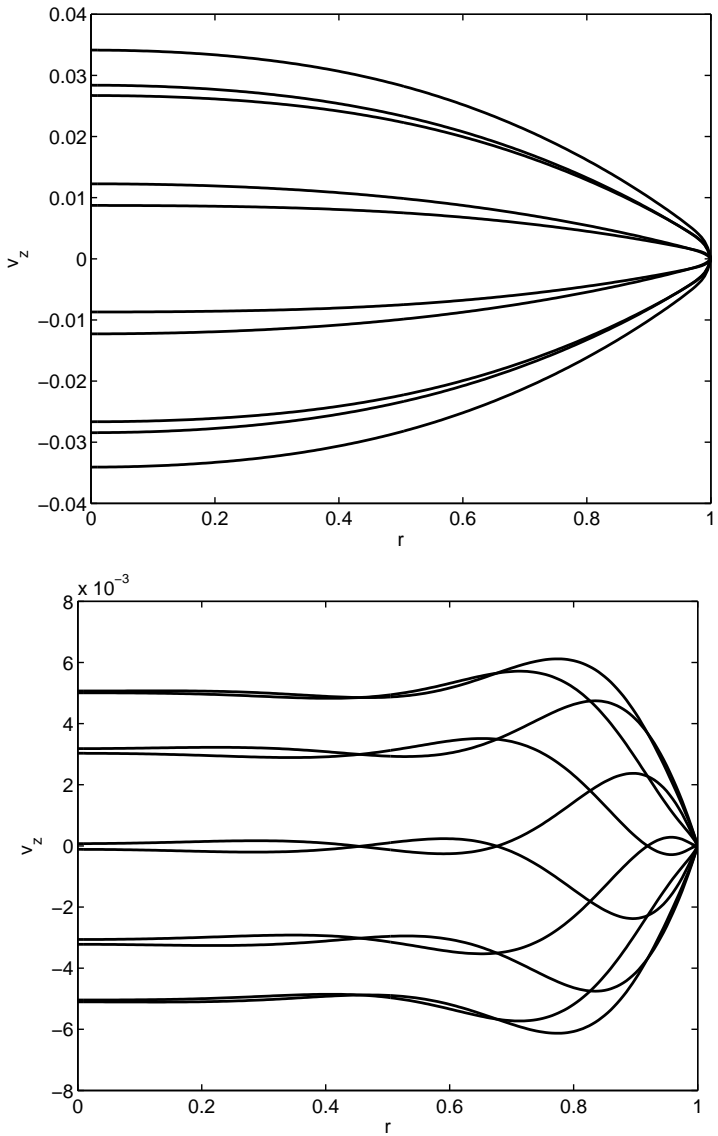

(b)

Figure 3: $R=430 \mu m$. Dimensionless axial velocity profiles at times $t=0, T / 10, \ldots, 9 T / 10$. (a) $\omega=4 \pi \mathrm{rad} s^{-1}$, (b) $\omega=400 \pi \mathrm{rads}^{-1}$. (Reproduced from Figs. 9(a) and 11(a) of M. A. Moyers-Gonzalez et al. (2008), J. Non-Newtonian Fluid Mech. 155:161-173, with permission. (C)2008 Elsevier B.V.) 
plotted against the infinite shear rate Deborah number $D e_{\infty}$. The results show that $\sigma_{R}<0$ for all cases considered, even though for large tubes $\sigma_{R} \rightarrow 0^{-}$as $D e_{\infty}$ becomes large. This gives us grounds for hoping that any (small) numerical differences between the solutions to the time-dependent equations (55), (65), (67), (68) and the steady values $N_{s}, M_{s}, \tau_{s}$ and $\sigma_{s}$ when the time dependent equation set is solved subject to the boundary conditions detailed in Appendix A and with initial conditions the steady variable values will eventually decay to zero. It should be noted, however, that the linear stability analysis performed here, only predicts the long-term behaviour of infinitesimal disturbances to the steady variables $N_{s}, M_{s}, \tau_{s}$ and $\sigma_{s}$. Note, from (87) that

$$
\boldsymbol{D}^{-1}\left(\mathcal{L}-\boldsymbol{P}^{-1} \boldsymbol{C P}\right)
$$

where $\boldsymbol{D}=\operatorname{diag}(2,1,2,1)$, is non-normal and that therefore, although its spectrum is predicted, for all values of $D e_{\infty}$ and $R$, to lie in the negative real halfplane, transient growth of infinitesimal disturbances is theoretically possible, so that, depending upon the value of $D e_{\infty}$ and the accuracy of the numerical method, these disturbances may grow and begin to interact in a non-linear fashion, possibly driving the flow unstable. See, for example, [3, 22, 40] for a detailed discussion of the consequences of non-normality upon the stability of flows. In the present case no such behaviour was observed, however, and, accordingly, the leading order solutions from the multiple time scales analysis described in Section 4.2 were set equal to their steady values.

In Figs. 5 and 6 we show, for $\omega=4 \pi \operatorname{rad} s^{-1}$ and $\omega=400 \pi \mathrm{rad} s^{-1}$, $\operatorname{respec}-$ tively, the leading order dimensionless value $N_{s} D e_{s}$ of the polymeric viscosity $N_{0} D e$ as well as profiles of the full dimensionless numerical solution. The profiles of $N_{0} D e$ are shown at ten equally spaced points in time $t=0, T / 10, \ldots, 9 T / 10$ throughout a full cycle (of period $T$ ). The tube radius is equal to $100 \mu \mathrm{m}$. Since, at the higher frequency the underlying microstructure does not have as much time to respond to changes in the local shear rate, variation in time of $N_{0} D e$ in Fig. 6(b) is smaller than that shown in Fig. 5(a). Convergence towards the steady leading order profile is clear as $\omega$ increases. Note that the steady leading order solutions $N_{s}, M_{s}, \sigma_{s}, \tau_{s}$ are independent of $t$ and $\vartheta$ but do change with $\omega$. This is because we fix the flow rate amplitude $P_{M}$ in all our simulations which results, as explained in Section 5.2 in a volume flow rate amplitude which varies with $\omega$. Particularly simple expressions for the leading order variables result in the homogeneous ( $\mathrm{Pe} \rightarrow \infty$ or infinite radius tube) case. Here the dimensionless variables $N_{0}=N_{s}=1$ and $\tau_{r r}=\tau_{s}=0$. The homogeneous analogue of (71) is then just

$$
\left(M_{s}\right)^{2} \frac{\langle a\rangle}{2}-\left(N_{s}-M_{s}\right) \frac{\langle b\rangle}{2}=0
$$

so that, writing the steady leading order average aggregate size as $n_{s}=1 / M_{s}$ we get

$$
n_{s}\left(n_{s}-1\right)=\frac{\langle a\rangle}{\langle b\rangle} .
$$

In Figs. 7(a) and (b) we demonstrate the convergence, for both the homogeneous model and the non-homogeneous model, of the physical average aggregate size 


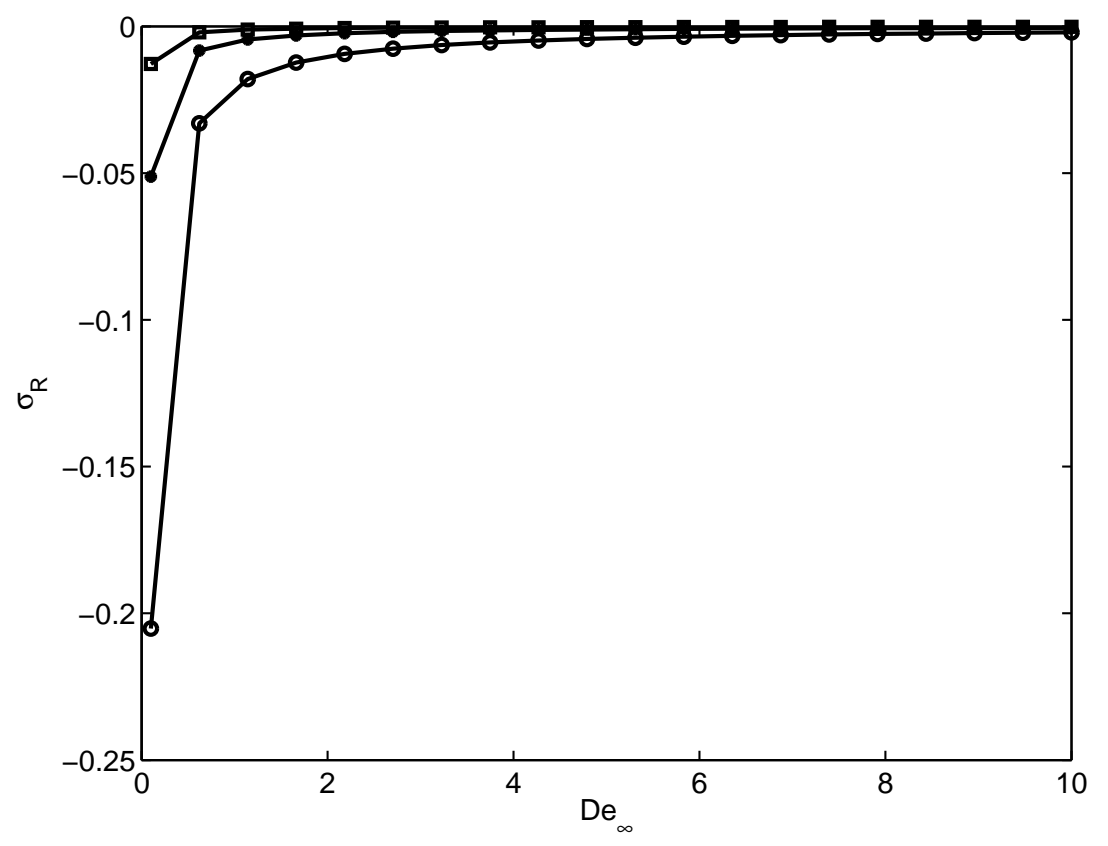

Figure 4: Maximum real part $\sigma_{R}$ of leading eigenvalue of the generalized eigenvalue problem (84). ॰: $R=50 \mu m, \bullet: R=100 \mu m, \square: R=200 \mu m$. 
(a)

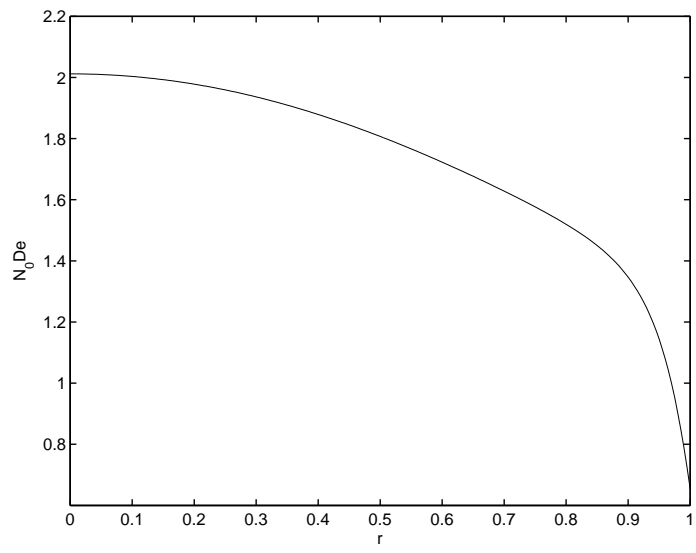

(b)

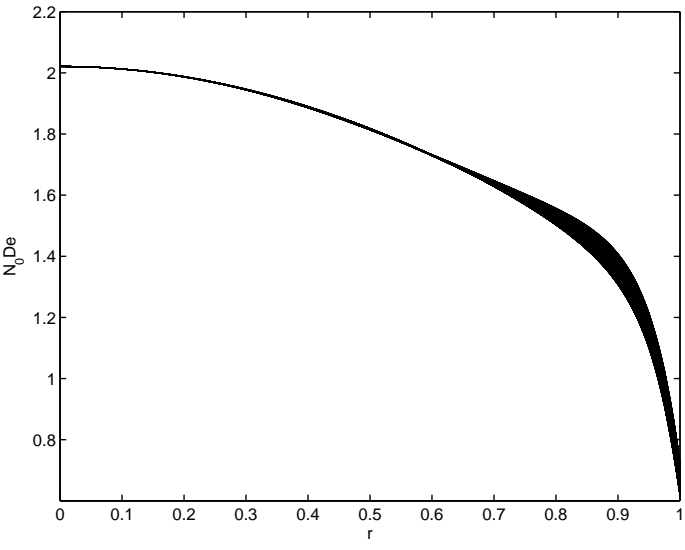

(c)

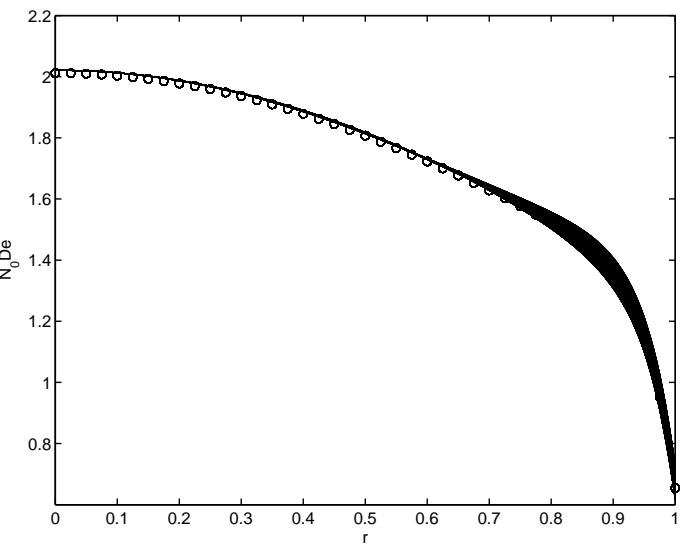

Figure 5: $R=100 \mu m, \omega=4 \pi \mathrm{rad}^{-1}$. (a) $N_{s} D e_{s}, \quad$ (b) $N_{0} D e$ at times $t=$ $0, T / 10, \ldots, 9 T / 10,(\mathrm{c})$ superposition of (a) and (b). 
(a)

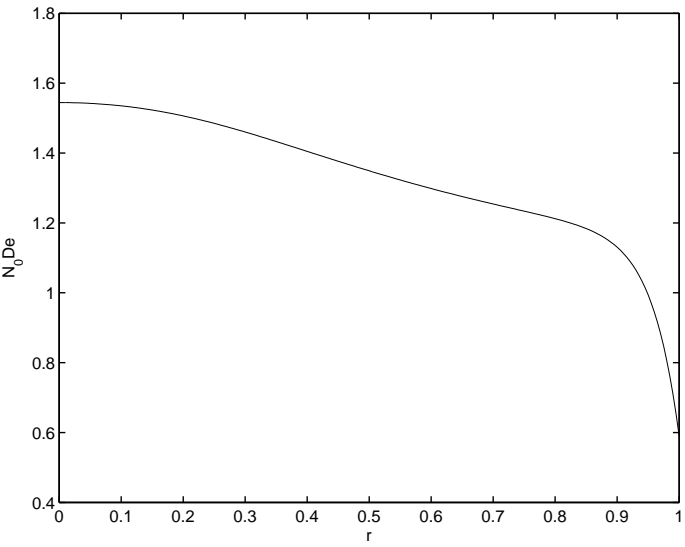

(b)

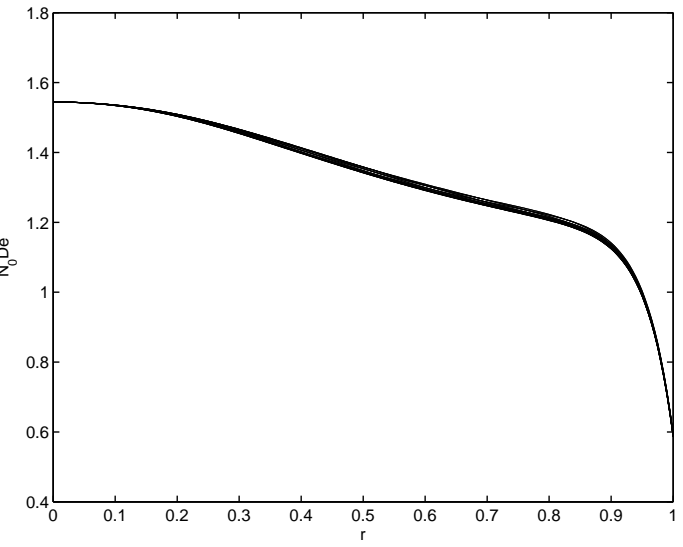

(c)

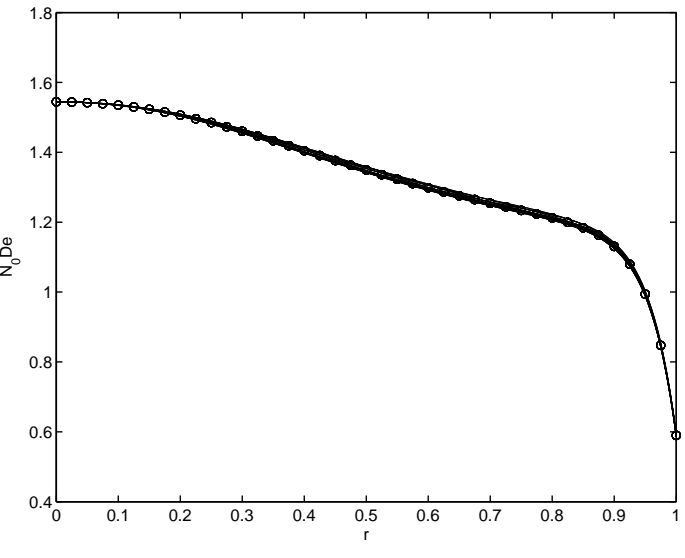

Figure 6: $R=100 \mu m, \omega=400 \pi \mathrm{rad}^{-1}$. (a) $N_{s} D e_{s}, \quad$ (b) $N_{0} D e$ at times $t=$ $0, T / 10, \ldots, 9 T / 10$, (c) superposition of (a) and (b). 

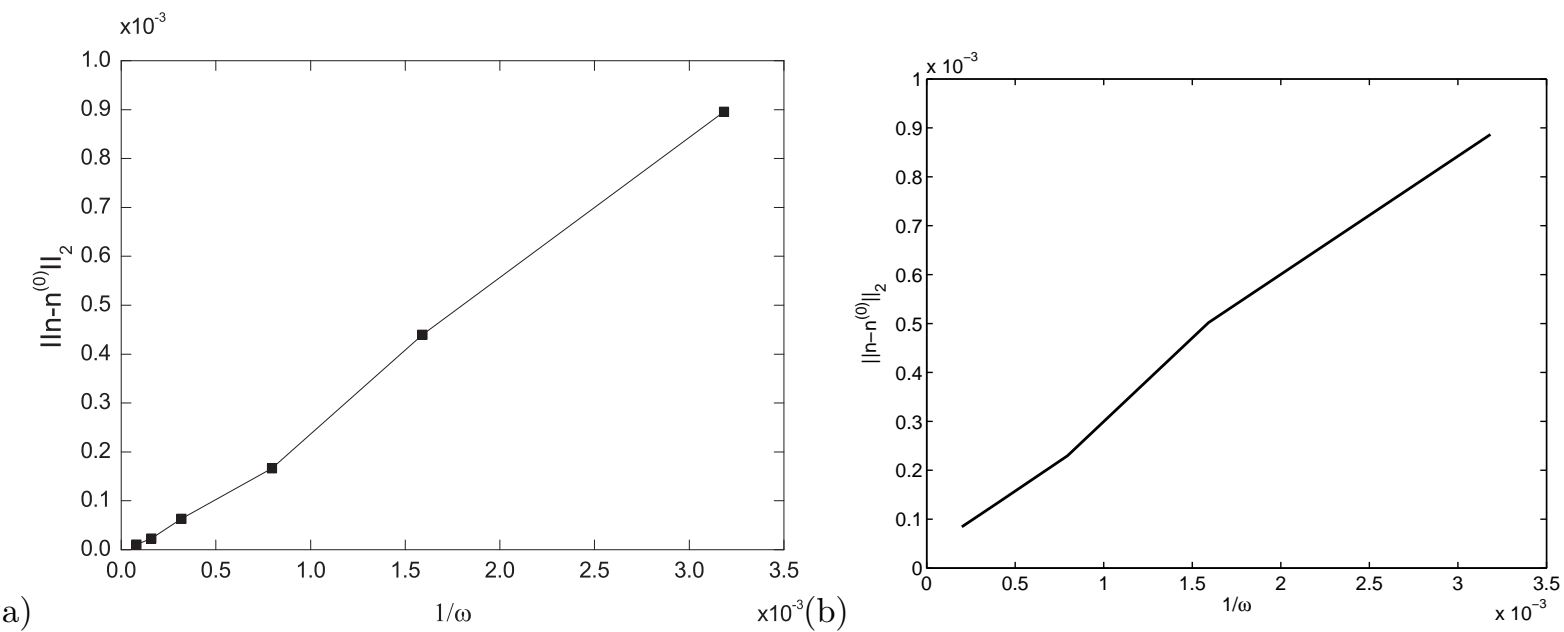

Figure 7: Maximum over one cycle of $\left\|n_{s}-n\right\|_{2}$. (a) Homogeneous model of [16, 29], (b) $R=100 \mu m$, non-homogeneous model.

to the leading order multiple time scales values as $\omega$ increases. Shown in both graphs is the the maximum over one complete cycle of the $L_{2}$ norm of the difference in the two variables and the convergence rate appears to be very similar in the two cases. If $n$ is written out in a multiple time scales expansions (cf. (40)-(44)) as

$$
n=n_{s}+\varepsilon n^{(1)}+\varepsilon^{2} n^{(2)}+\ldots,
$$

it follows that the gradient of the curves in Figs. $7(\mathrm{a})$ and (b) as $1 / \omega \rightarrow 0$ tends to

$$
\max _{t \in[0, T]}\left\|n^{(1)}\right\|_{2} .
$$

Convergence with increasing values of the frequency is also clear from Fig. 8 (a) and (b) where we show the results of computing the maximum $L_{2}$ norm of the difference between $M$ and $M_{s}$ and between $N_{0}$ and $N_{s}$. Were we to show the corresponding results for $\tau_{r r}$ they would similarly show convergence to $\tau_{s}$ as $1 / \omega \rightarrow 0$.

\section{Conclusions}

The high frequency behaviour of healthy human blood in rapid oscillatory tube flow has been described via computations of the pressure gradient amplitude - volume flow rate amplitude relationship and via a multiple time scales analysis of the governing equations of the hemorheological model of MoyersGonzalez et al. [25, 26, 27].

The pressure gradient amplitude - volume flow rate amplitude results in a tube of radius $430 \mu \mathrm{m}$ and at an oscillatory frequency of $\omega=2 \mathrm{~Hz}$ have been 

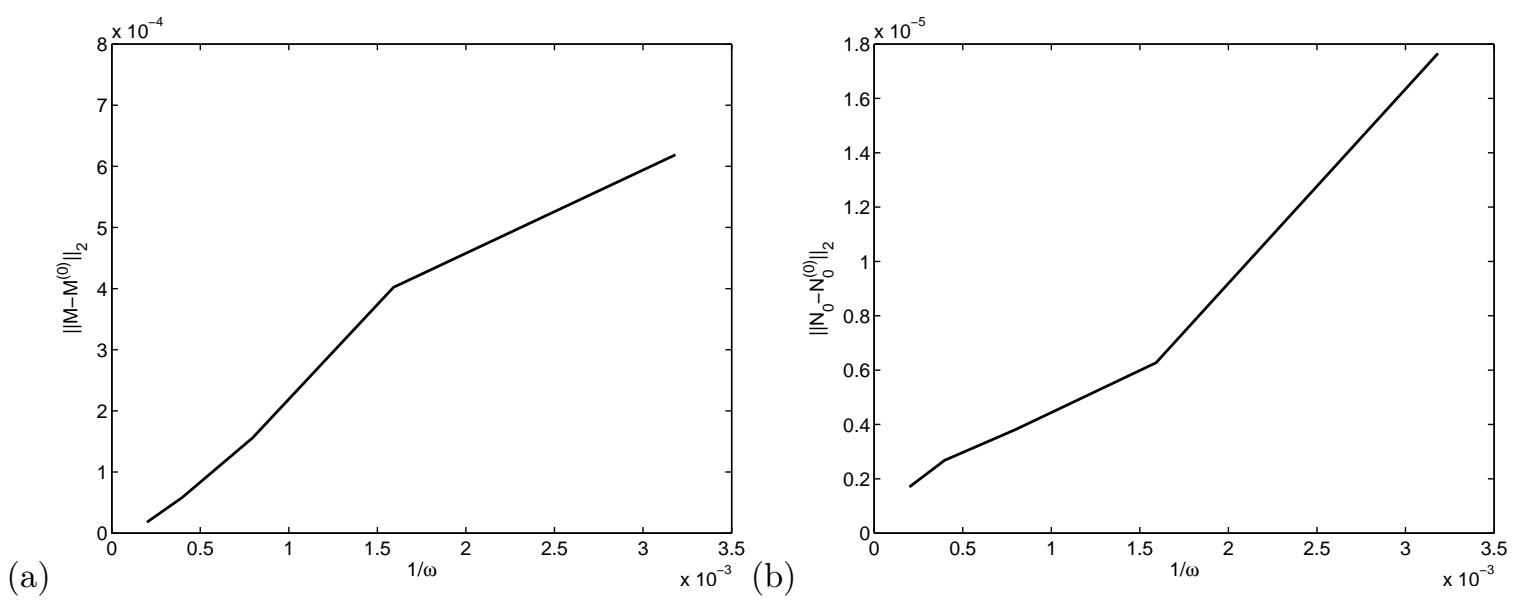

Figure 8: $R=100 \mu m$. (a) Maximum over one cycle of $\left\|M_{s}-M\right\|_{2}$, (b) Maximum over one cycle of $\left\|N_{s}-N_{0}\right\|_{2}$.

shown to be in excellent agreement with the experimental data of Thurston [38]. Allowing the oscillatory frequency to increase beyond far beyond $2 \mathrm{~Hz}$ we have then presented evidence of the existence of a single resonant frequency $\omega_{r}$ at which the volume flow rate is maximized at a fixed pressure gradient amplitude. For frequencies higher than $\omega_{r}$ the pressure gradient amplitude required to maintain a constant volume flow rate must be continuously increased with increasing oscillatory frequency. The existence of a resonant frequency in oscillatory tube flow of viscoelastic fluids is well documented and may be present even for linear viscoelastic fluids. It is important to distinguish resonance from flow enhancement in pulsatile flows, however, where any increase in the time-averaged flow rate relative to its value at zero frequency is a non-linear viscoelastic effect [2], although this mechanism might be most effective near resonant frequencies.

A key outcome of the multiple time scales analysis has been that the red cell number density, average aggregate size and $r r$-component of the elastic stress are all functions of the radius alone, to leading order. A consequence of this is that the equation of linear momentum for blood undergoing rapid oscillatory flow is predicted to be that of a generalized linear viscoelastic fluid (114) with an $r$-dependent complex viscosity (126).

Our demonstration of generalized linear viscoelastic behaviour in our nonhomogeneous blood model when volume flow rate amplitudes are small or oscillatory frequencies very high has enabled us to interpret the computed results in these cases. For example, we have shown, at least over a certain range of oscillatory frequencies, that the phase shift $A$ (see (5)) of the volume flow rate oscillations relative to those of the pressure gradient passes from positive (more viscoelastic behaviour) to negative (more viscous behaviour) as the volume flow rate amplitude increases. At some oscillatory frequencies and at critical values 
of the volume flow rate amplitude the volume flow rate and the pressure gradient might be seen to oscillate perfectly in phase and this has been shown to be caused by a phase shift due to elasticity cancelling that due to inertia. As oscillatory frequencies grow to be sufficiently large the fluid behaves more and more like an elastic solid in the sense that $A \rightarrow \pi / 2$, although what is really happening is that the inertial term $\hat{\omega} R e / \pi$ dominates over all others in (24)-(25) so that, despite the fact that the elastic component $\eta^{\prime \prime}$ of the complex viscosity tends to zero and the fluid therefore becomes increasingly Newtonian, the stress becomes proportional to the strain rather than to the strain rate.

\section{Acknowledgements}

The second author would like to thank Marco Marletta (Cardiff University), Dmitry Pelinovsky (McMaster University) and Jie Shen (Purdue University) for useful discussions and correspondence on the subject of the eigenspectrum of the generalized eigenvalue problem (84).

\section{Appendix A: Initial and boundary conditions for (28)-(34)}

For a well-posed problem we need to prescribe suitable initial and boundary conditions for the coupled system of equations (28)-(34). For all computations performed in this paper the initial conditions were chosen to the solutions of the steady Poiseuille problem with $\partial p / \partial z=-P_{M}$. Elastic stress boundary conditions have been derived from the assumption (see, too, $[5,10,33])$ that all microstructures on the wall lie with their principal axes aligned in the $z$ direction. From the Kramers [20] expression for the elastic stress tensor this means that

$$
\boldsymbol{\tau}(1, t)=N_{0}(1, t) Q_{0}^{2} \boldsymbol{e}_{z} \boldsymbol{e}_{z}-N_{0}(1, t) \boldsymbol{\delta},
$$

where, $Q_{0}$ is a scaled dumbbell length and $\boldsymbol{e}_{z}$ is a unit vector in the $z$-direction. Fortunately, and as remarked in Section 4.1 we have no need of the $z z$-component of $\boldsymbol{\tau}$ in order to compute the other variables. Thus, it is unnecessary to estimate $Q_{0}$ in the Kramers expression above and the wall boundary condition for the remaining components of $\boldsymbol{\tau}$ is just

$$
\tau_{i j}(1, t)=-N_{0}(1, t) \delta_{i j} .
$$

The boundary condition on $r=1$ for the axial component of the velocity is that of the no-slip condition:

$$
v_{z}(1, t)=0,
$$

whereas the wall conditions for $N_{0}$ and $M$ are the natural boundary conditions

$$
\frac{\partial N_{0}}{\partial r}(1, t)-\frac{\partial \tau_{r r}}{\partial r}(1, t)=0 \text { and } \frac{\partial M}{\partial r}(1, t)-\frac{\partial \sigma_{r r}}{\partial r}(1, t)=0 .
$$

The above wall condition on $N_{0}$ is equivalent to ensuring that there is no flux of rouleaux through the tube walls. 
Along the axis of symmetry $r=0$ shear-free and symmetry conditions are imposed on all variables except $N_{0}$, leading to

$$
\tau_{r z}(0, t)=0, \frac{\partial v_{z}}{\partial r}(0, t)=0, \frac{\partial M}{\partial r}(0, t)=0 \text { and } \frac{\partial \tau_{r r}}{\partial r}(0, t)=0 .
$$

Finally, a non-local condition integral condition,

$$
\int_{r=0}^{1} N_{0} r d r=\frac{1}{2}
$$

meaning that the number of dumbbells in any cylindrical section of the tube is a constant, is imposed on $N_{0}$. In fact, it should be borne in mind that what appears in (100) is the dimensionless $N_{0}$. The same volume integral of the physical (dimensional) $N_{0}$ would not be a constant, but vary with the tube radius, due to particle migration (the Fåhraeus effect, [14]).

\section{Appendix B: Zeroth order Deborah number $D e^{(0)}$}

From (38) we may write down the zeroth order term in the multiple time scales expansion (44) for $D e$ as follows:

$$
D e^{(0)}=\frac{n^{(0)} D e_{\infty}}{1+g_{n^{(0)}} n^{(0)} D e_{\infty}}
$$

where $n^{(0)}=N_{0}^{(0)} / M^{(0)}$ is the zeroth order average aggregate size and

$$
g_{n^{(0)}} n^{(0)}=\frac{b}{2} n^{(0)}\left(n^{(0)}-1\right)+a N_{0}^{(0)} .
$$

Therefore, the zeroth order Deborah number $D e^{(0)}$ becomes

$$
D e^{(0)}=\frac{N_{0}^{(0)} M^{(0)} D e_{\infty}}{M^{(0)^{2}}+\left(\frac{b}{2} N_{0}^{(0)}\left(N^{(0)}-M^{(0)}\right)+a N_{0}^{(0)} M^{(0)^{2}}\right) D e_{\infty}} .
$$

We thus have

$$
D e^{(0)}=\frac{\left(N_{s} M_{s}+\epsilon\left(N_{1}^{(0)} M_{s}+N_{s} M_{1}^{(0)}\right)+O\left(\epsilon^{2}\right)\right) D e_{\infty}}{A+B \epsilon+O\left(\epsilon^{2}\right)},
$$

where the functions $A$ and $B$ appearing in the denominator of (104) are given by

$$
A=M_{s}^{2}+\left(\frac{b}{2} N_{s}\left(N_{s}-M_{s}\right)+a N_{s} M_{s}^{2}\right) D e_{\infty}
$$


and

$$
\begin{aligned}
B=2 M_{s} & M_{1}^{(0)} \\
& \\
& +\left(\frac{b}{2} N_{s}\left(N_{1}^{(0)}-M_{1}^{(0)}\right)+\frac{b}{2} N_{1}^{(0)}\left(N_{s}-M_{s}\right)+2 a N_{s} M_{s} M_{1}^{(0)}\right. \\
& \left.\quad+a N_{1}^{(0)} M_{s}^{2}\right) D e_{\infty} .
\end{aligned}
$$

Thus, using the binomial theorem to expand the denominator we get

$$
\begin{aligned}
D e^{(0)} & =\frac{D e_{\infty}}{A}\left(N_{s} M_{s}+\epsilon\left(N_{1}^{(0)} M_{s}+N_{s} M_{1}^{(0)}\right)+O\left(\epsilon^{2}\right)\right)\left(1-\epsilon \frac{B}{A}+O\left(\epsilon^{2}\right)\right), \\
& =D e_{s}+\epsilon D e_{1}+O\left(\varepsilon^{2}\right),
\end{aligned}
$$

where

$$
D e_{s}=\frac{D e_{\infty} N_{s} M_{s}}{M_{s}^{2}+\left(\frac{b}{2} N_{s}\left(N_{s}-M_{s}\right)+a N_{s} M_{s}^{2}\right) D e_{\infty}},
$$

and

$$
\begin{aligned}
& D e_{1}= \frac{D e_{s}}{N_{s} M_{s}}\left(N_{1}^{(0)} M_{s}+N_{s} M_{1}^{(0)}\right) \\
&-\frac{D e_{s}^{2}}{D e_{\infty} N_{s} M_{s}}\left(2 M_{s} M_{1}^{(0)}+\frac{b}{2} D e_{\infty}\left(N_{s}\left(N_{1}^{(0)}-M_{1}^{(0)}\right)+N_{1}^{(0)}\left(N_{s}-M_{s}\right)\right)\right. \\
&\left.\quad+a D e_{\infty}\left(2 N_{s} M_{s} M_{1}^{(0)}+N_{1}^{(0)} M_{s}^{2}\right)\right)
\end{aligned}
$$

Thus, writing $D e_{1}=\widehat{D e} \exp (\lambda t), N_{1}^{(0)}=\widehat{N} \exp (\lambda t)$ and $M_{1}^{(0)}=\widehat{M} \exp (\lambda t)$ and computing the $\vartheta$-cyclical average $\langle\cdot\rangle$ of all terms, we conclude that

$$
\begin{aligned}
\frac{\widehat{D e}}{D e_{s}^{2}} & =\frac{\hat{N}}{N_{s} M_{s}}\left(\frac{M_{s}}{D e_{s}}-\left(\frac{b}{2}\left(2 N_{s}-M_{s}\right)+a M_{s}^{2}\right)\right) \\
& +\frac{\hat{M}}{N_{s} M_{s} D e_{\infty}}\left(\frac{N_{s} D e_{\infty}}{D e_{s}}-2 M_{s}+D e_{\infty}\left(\frac{b}{2} N_{s}-2 a N_{s} M_{s}\right)\right), \\
\Rightarrow\left\langle\frac{\widehat{D e}}{D e_{s}^{2}}\right\rangle & =\alpha \hat{N}+\beta \hat{M}
\end{aligned}
$$

where the functions $\alpha=\alpha(r), \beta=\beta(r)$ are given by

$$
\alpha=\frac{1}{N_{s} M_{s}}\left(M_{s}\left\langle\frac{1}{D e_{s}}\right\rangle-\left(\frac{\langle b\rangle}{2}\left(2 N_{s}-M_{s}\right)+\langle a\rangle M_{s}^{2}\right)\right),
$$


and

$$
\beta=\frac{1}{N_{s} M_{s} D e_{\infty}}\left(N_{s} D e_{\infty}\left\langle\frac{1}{D e_{s}}\right\rangle-2 M_{s}+D e_{\infty}\left(\frac{\langle b\rangle}{2} N_{s}-2\langle a\rangle N_{s} M_{s}\right)\right) .
$$

\section{Appendix C: Generalization of (10) for the blood model of Moyers- Gonzalez et al. [25, 26, 27]}

In this Appendix we seek to show that an axial velocity field $v_{z}$ of the complex form

$$
v_{z}=v_{z}^{*}(r) \exp (i \hat{\omega} t)
$$

(see (39)) may be obtained from the non-homogeneous hemorheological model of Moyers-Gonzalez et al. [25, 26, 27], when $\hat{\omega} \gg 1$, by imposing an oscillatory pressure gradient (3), having the same oscillatory frequency, albeit with a phase shift. This would certainly be true if $v_{z}$ were to satisfy the equation of motion of a generalized linear viscoelastic model

$$
R e \frac{\partial v_{z}}{\partial t}-\frac{1}{r} \frac{d}{d r}\left(r \eta^{*}(r) \frac{\partial v_{z}}{\partial r}\right)=P_{M} \exp (i \hat{\omega} t)
$$

with an $r$-dependent complex viscosity $\eta^{*}$ since, in this case, we would only have to solve a two-point boundary value problem for $v_{z}^{*}$

$$
i \hat{\omega} R e v_{z}^{*}-\frac{1}{r} \frac{d}{d r}\left(r \eta^{*}(r) \frac{d v_{z}^{*}}{d r}\right)=P_{M}
$$

subject to the boundary conditions

$$
v_{z}^{*}(1)=0 \text { and } \frac{\partial v_{z}^{*}}{\partial r}(0)=0 .
$$

We take the remainder of this Appendix to show that $v_{z}$ of the form (113) leads to a well-defined complex viscosity $\eta^{*}$ in $(114)$, at least up to $O(1 / \hat{\omega})$. This necessitates determining the shear stress-velocity gradient relationship.

To this end, we write out the elastic shear stress $\tau_{r z}$ in a multiple time scales expansion thus:

$$
\tau_{r z}=\tau_{r z}^{(0)}(r, t, \vartheta)+\varepsilon \tau_{r z}^{(1)}(r, t, \vartheta)+\varepsilon^{2} \tau_{r z}^{(2)}(r, t, \vartheta)+\ldots
$$

Then, using the expansions (40), (41), (44) and (117) in the shear stress equation 
(31) and supposing the axial velocity $v_{z}$ to be of the form (113), we obtain

$$
\begin{gathered}
\left(D e^{(0)}+\varepsilon D e^{(1)}+\varepsilon^{2} D e^{(2)}+\ldots\right)\left[\left(\frac{\partial \tau_{r z}^{(0)}}{\partial t}+\frac{1}{\varepsilon} \frac{\partial \tau_{r z}^{(0)}}{\partial \vartheta}\right)+\varepsilon\left(\frac{\partial \tau_{r z}^{(1)}}{\partial t}+\frac{1}{\varepsilon} \frac{\partial \tau_{r z}^{(1)}}{\partial \vartheta}\right)\right. \\
\left.+\varepsilon^{2}\left(\frac{\partial \tau_{r z}^{(2)}}{\partial t}+\frac{1}{\varepsilon} \frac{\partial \tau_{r z}^{(2)}}{\partial \vartheta}\right)+\ldots\right] \\
-\left(D e^{(0)}+\varepsilon D e^{(1)}+\varepsilon^{2} D e^{(2)}+\ldots\right) \frac{1}{P e}\left(\frac{1}{r} \frac{\partial}{\partial r}\left[r\left(\frac{\partial \tau_{r z}^{(0)}}{\partial r}+\varepsilon \frac{\partial \tau_{r z}^{(1)}}{\partial r}+\varepsilon^{2} \frac{\partial \tau_{r z}^{(2)}}{\partial r}+\ldots\right)\right]\right. \\
\left.\quad-\frac{1}{r^{2}}\left(\tau_{r z}^{(0)}+\varepsilon \tau_{r z}^{(1)}+\varepsilon^{2} \tau_{r z}^{(2)}+\ldots\right)\right) \\
-\left(\tau_{r r}^{(0)}+\varepsilon \tau_{r r}^{(1)}+\varepsilon^{2} \tau_{r r}^{(2)}+\ldots+N_{0}^{(0)}+\varepsilon N_{0}^{(1)}+\varepsilon^{2} N_{0}^{(2)}+\ldots\right) \\
\times\left(D e^{(0)}+\varepsilon D e^{(1)}+\varepsilon^{2} D e^{(2)}+\ldots\right) \frac{\partial v_{z}}{\partial r} \\
+\left(\tau_{r z}^{(0)}+\varepsilon \tau_{r z}^{(1)}+\varepsilon^{2} \tau_{r z}^{(2)}+\ldots\right)=0 .
\end{gathered}
$$

From considering the $O\left(\varepsilon^{-1}\right)$ term we deduce immediately that $\tau_{r z}^{(0)}$ is independent of the fast time $\vartheta$. Equating all $O(1)$ terms leads to

$$
\begin{aligned}
D e^{(0)} \frac{\partial \tau_{r z}^{(0)}}{\partial t}+D e^{(0)} \frac{\partial \tau_{r z}^{(1)}}{\partial \vartheta} & -\frac{D e^{(0)}}{P e}\left(\frac{1}{r} \frac{\partial}{\partial r}\left(r \frac{\partial \tau_{r z}^{(0)}}{\partial r}\right)-\frac{\tau_{r z}^{(0)}}{r^{2}}\right) \\
& -\left(\tau_{r r}^{(0)}+N_{0}^{(0)}\right) D e^{(0)} \frac{\partial v_{z}}{\partial r}+\tau_{r z}^{(0)}=0 .
\end{aligned}
$$

Define

$$
Q:=\frac{\partial \tau_{r z}^{(0)}}{\partial t}-\frac{1}{P e}\left(\frac{1}{r} \frac{\partial}{\partial r}\left(r \frac{\partial \tau_{r z}^{(0)}}{\partial r}\right)-\frac{\tau_{r z}^{(0)}}{r^{2}}\right)-\left(\tau_{r r}^{(0)}+N_{0}^{(0)}\right) \frac{\partial v_{z}}{\partial r}+\frac{1}{D e^{(0)}} \tau_{r z}^{(0)} .
$$

Then, since all variables appearing on the right-hand side of (120) are either independent of $\vartheta\left(\tau_{r z}^{(0)}, \tau_{r r}^{(0)}, N_{0}^{(0)}\right)$ or, when dependent on $\vartheta$, are $2 \pi$-periodic $\left(\partial v_{z} / \partial r, D e^{(0)}\right)$, we may write $Q$ in the form

$$
Q:=\langle Q\rangle+G,
$$

where $G$ represents the part of $Q$ that oscillates about $\langle Q\rangle$. We may now argue, as in the case of $R$ and $S$ in Section 4.2, to conclude that $\langle Q\rangle=0$. That is,

$$
\frac{\partial \tau_{r z}^{(0)}}{\partial t}-\frac{1}{P e}\left(\frac{1}{r} \frac{\partial}{\partial r}\left(r \frac{\partial \tau_{r z}^{(0)}}{\partial r}\right)-\frac{\tau_{r z}^{(0)}}{r^{2}}\right)+\left\langle\frac{1}{D e^{(0)}}\right\rangle \tau_{r z}^{(0)}=0,
$$

where we have used the fact that $v_{z}$ of the form (39) has zero cyclical average. i.e.

$$
\left\langle\frac{\partial v_{z}}{\partial r}\right\rangle=0
$$


The solution to (121), subject to zero Dirichlet conditions on $r=0$ and $r=1$ and zero initial conditions, is just $\tau_{r z}^{(0)}=0$. Therefore, from (119) the equation for $\tau_{r z}^{(1)}$ to be solved, subject to zero Dirichlet conditions on $r=0$ and $r=1$ is

$$
\frac{\partial \tau_{r z}^{(1)}}{\partial \vartheta}=\left(\tau_{r r}^{(0)}+N_{0}^{(0)}\right) \frac{\partial v_{z}}{\partial r}
$$

which, integrating throughout with respect to $\vartheta$ and using the $r r$-component of (96) and the Neumann condition on $v_{z}$ in (99), yields

$$
\tau_{r z}^{(1)}=\frac{1}{i}\left(\tau_{r r}^{(0)}+N_{0}^{(0)}\right) \frac{\partial v_{z}}{\partial r} .
$$

Note that since we may take $\tau_{r r}^{(0)}=\tau_{s}$ and $N_{0}^{(0)}=N_{s}$ (see Section 4.3), the simple linear relation (73) and the $r r$ - component of (96) imply that (124) may be rewritten as

$$
\tau_{r z}^{(1)}=\frac{2}{i}\left(N_{s}(r)-N_{s}(1)\right) \frac{\partial v_{z}}{\partial r} .
$$

Using (125) we may now write down the complex viscosity $\eta^{*}$ appearing in (114) in terms of its real and imaginary parts as

$$
\eta^{*}\left(=\eta^{\prime}-i \eta^{\prime \prime}\right)=\eta-2 i \varepsilon\left(N_{s}(r)-N_{s}(1)\right)+O\left(\varepsilon^{2}\right)
$$

where $\eta$ is the (constant) plasma viscosity.

\section{References}

[1] The American Heart Association, Target Heart Rates, http://www.americanheart.org.

[2] Y. A. Andrienko, D. A. Siginer and Y. G. Yanovsky, Resonance behavior of viscoelastic fluids in Poiseuille flow and application to flow enhancement, Int. J. Non-Linear Mech. 35 (2000) 95-102.

[3] K. Atalık and R. Keunings, Non-linear temporal stability analysis of viscoelastic plane channel flows using a fully-spectral method, J. NonNewtonian Fluid Mech. 102 (2002) 299-319.

[4] H. A. Barnes, J. F. Hutton and K. Walters, An Introduction to Rheology, Elsevier, Amsterdam, (1989).

[5] A. V. Bhave, R. C. Armstrong and R. A. Brown, Kinetic theory and rheology of dilute, non-homogeneous polymer solutions, J. Chem. Phys. 95 (1991) 2988-3000.

[6] L. J. F. Broer, On the hydrodynamics of viscoelastic fluids, Appl. Sci. Res. A6 (1957) 226-236. 
[7] M. Bureau, J. C. Healy, D. Bourgoin and M. Joly, Rheological hysteresis of blood at low shear rate, Biorheology 17 (1980) 191-203.

[8] J. R. Castrejón-Pita, J. A. del Río, A. A. Castrejón-Pita and G. Huelsz, Experimental observation of dramatic differences in the dynamic response of Newtonian and Maxwellian fluids, Phys. Rev. E 68 (2003) 046301-1046301-5.

[9] S. Chien, S. Usami and R. Skalak, Blood flow in small tubes, in S. R. Geiger (ed.) Handbook of Physiology, Section 2: The Cardiovascular System, Volume IV Part 1. Bethesda, Md, American Physiological Society, 1984, pp. $217-249$.

[10] L. P. Cook and L. F. Rossi, Slippage and migration in models of dilute wormlike micellar solutions and polymeric fluids, J. Non-Newtonian Fluid Mech. 116 (2004) 347-369.

[11] N. A. Coulter Jr. and M. Singh M, Frequency dependence of blood viscosity in oscillatory flow, Biorheology 8 (1971) 115-124.

[12] W. P. Cox and E. H. Merz, Correlation of dynamic and steady flow viscosities, J. Polym. Sci. 28 (1958) 619-622.

[13] J. A. del Río, M. López de Haro and S. Whitaker, Enhancement in the dynamic response of a viscoelastic fluid flowing in a tube, Phys. Rev. E 58 (1998) 6323-6327. (Erratum: Phys. Rev. E. 64 (2001) 039901.)

[14] R. Fåhraeus, The suspension stability of the blood, Physiol. Rev. 9 (1929) $241-274$.

[15] R. Fåhraeus and T. Lindqvist, The viscosity of blood in narrow capillary tubes, Am. J. Physiol. 96 (1931) 562-568.

[16] J. Fang and R. G. Owens, Numerical simulations of pulsatile blood flow using a new constitutive model, Biorheology 43 (2006) 637-660.

[17] A. G. Fredrickson, Principles and applications of rheology, Prentice-Hall, Englewood Cliffs, NJ, (1964).

[18] D. L. Fry, D. M. Griggs and J. C. Greenfield, In vivo studies of pulsatile blood flow: The relationship of the pressure gradient to the blood velocity. In: E. O. Attinger (ed.), Pulsatile blood flow. McGraw-Hill, New York, (1964).

[19] P. B. Kahn PB, Mathematical methods for scientists and engineers: linear and nonlinear systems, John Wiley \& Sons, New York, (1990).

[20] H. A. Kramers, Het gedrag van macromoleculen in een stroomende vloeistof, Physica 11 (1944) 1-19. 
[21] A. L. Kunz and N. A. Coulter Jr., Non-Newtonian behavior of blood in oscillatory flow, Biophys. J. 7 (1967) 25-36.

[22] R. Kupferman, On the linear stability of plane Couette flow for an OldroydB fluid and its numerical approximation, J. Non-Newtonian Fluid Mech. 127 (2005) 169-190.

[23] M. A. Moyers-Gonzalez, Transient effects in oilfield cementing flows, Phd Thesis, University of British Columbia, (2006).

[24] M. A. Moyers-Gonzalez and I. A. Frigaard, Kinematic instabilities in twolayer eccentric annular flows, part 1: Newtonian fluids, J. Eng. Maths 62 (2008) 103-131.

[25] M. A. Moyers-Gonzalez, R. G. Owens and J. Fang, A non-homogeneous constitutive model for human blood. Part I: Model derivation and steady flow, J. Fluid Mech. 617 (2008) 327-354.

[26] M. A. Moyers-Gonzalez and R. G. Owens, A non-homogeneous constitutive model for human blood. Part II: Asymptotic solution for large Péclet numbers, J. Non-Newtonian Fluid Mech. 155 (2008) 146-160.

[27] M. A. Moyers-Gonzalez, R. G. Owens and J. Fang, A non-homogeneous constitutive model for human blood. Part III: Oscillatory flow, J. NonNewtonian Fluid Mech. 155 (2008) 161-173.

[28] T. Murata and T. W. Secomb, Effects of shear rate on rouleau formation in simple shear flow, Biorheology 25 (1988) 113-122.

[29] R. G. Owens, A new microstructure-based constitutive model for human blood, J. Non-Newtonian Fluid Mech. 140 (2006) 57-70.

[30] A. R. Pries, D. Neuhaus and P. Gaehtgens, Blood viscosity in tube flow: dependence on diameter and hematocrit, Am. J. Physiol. Heart Circ. Physiol. 263 (1992) H1770-H1778.

[31] A. R. Pries, T. W. Secomb, P. Gaehtgens and J. F. Gross, Blood flow in microvascular networks. Experiments and simulation, Circ. Res. 67 (1990) 826-834.

[32] K. D. Rahaman and H. Ramkissoon, Unsteady axial viscoelastic pipe flows, J. Non-Newtonian Fluid Mech. 57 (1995) 27-38.

[33] L. F. Rossi, G. McKinley and L. P. Cook, Slippage and migration in TaylorCouette flow of a model for dilute wormlike micellar solutions, J. NonNewtonian Fluid Mech. 136 (2006) 79-92.

[34] P. Schmid and D. S. Henningson, Stability and transition in shear flows, Springer-Verlag, New York, (2001). 
[35] A. Siginer, On some nearly viscometric flows of viscoelastic liquids, Rheol. Acta 30 (1991) 447-473.

[36] G. B. Thurston, Theory of oscillation of a viscoelastic fluid in a circular tube, J. Acoust. Soc. Am. 32 (1960) 210-213.

[37] G. B. Thurston, Frequency and shear rate dependence of viscoelasticity of human blood, Biorheology 10 (1973) 375-381.

[38] G. B. Thurston, Elastic effects in pulsatile blood flow, Microvasc. Res. 9 (1975) 145-157.

[39] G. B. Thurston, The effects of frequency of oscillatory flow on the impedance of rigid, blood-filled tubes, Biorheology 13 (1976) 191-199.

[40] L. N. Trefethen, A. E. Trefethen, S. C. Reddy and T. A. Driscoll, Hydrodynamic stability without eigenvalues, Science 261 (1993) 578-584.

[41] D. Tsiklauri and I. Beresnev, Enhancement in the dynamic response of a viscoelastic fluid flowing through a longitudinally vibrating tube, Phys. Rev. E 63 (2001) 046304-1-4.

[42] H. Vandeven, On the eigenvalues of second-order spectral differentiation operators, Comput. Appl. Mech. Engrg. 80 (1990) 313-318.

[43] J. A. C. Weideman and L. N. Trefethen, The eigenvalues of second-order spectral differentiation matrices, SIAM J. Numer. Anal. 25 (1988) 12791298.

[44] J. R. Womersley, Method for the calculation of velocity, rate of flow and viscous drag in arteries when the pressure gradient is known, J. Physiol. 127 (1955) 553-563.

[45] J. R. Womersley, An elastic tube theory of pulse transmission and oscillatory flow in mammalian arteries, Wright Air Development Center Technical Report WADC-TR 56-614, Wright Air Development Center, Ohio, (1957).

[46] Y. Yin and K.-Q. Zhu, Oscillating flow of a viscoelastic fluid in a pipe with the fractional Maxwell model, Applied Mathematics and Computation 173 (2006) 231-242.

[47] M. Zamir, The physics of pulsatile flow, Springer-Verlag, New York, (2000). 